\title{
VERY AMPLENESS OF ADJOINT LINEAR SYSTEMS ON SMOOTH SURFACES WITH BOUNDARY
}

\author{
VLADIMIR MAŞEK
}

\begin{abstract}
Let $M$ be a $\mathbb{Q}$-divisor on a smooth surface over $\mathbb{C}$. In this paper we give criteria for very ampleness of the adjoint of $\lceil M\rceil$, the round-up of $M$. (Similar results for global generation were given by Ein and Lazarsfeld and used in their proof of Fujita's Conjecture in dimension 3.) In $\S 4$ we discuss an example which suggests that this kind of criteria might also be useful in the study of linear systems on surfaces.
\end{abstract}

\section{Notations}

$\lceil\cdot\rceil \quad$ round-up

$\lfloor\cdot\rfloor \quad$ round-down

$\{\cdot\} \quad$ fractional part

$f^{-1} D \quad$ strict transform (proper transform)

$f^{*} D \quad$ pull-back (total inverse image)

$P L C \quad$ partially log-canonical (Definition 1.7)

$\equiv \quad$ numerical equivalence

$\sim \quad$ linear equivalence

$\widetilde{\mathbb{Q}} \mathbb{Q}$-linear equivalence

\section{Introduction}

Let $S$ be a nonsingular projective surface over $\mathbb{C}$, and let $H$ be a given line bundle on $S$. Consider the following natural questions regarding the complete linear system $|H|$ :

(1) Compute $\operatorname{dim}|H|$.

(2) Is $|H|$ base-point-free?

Received August 21, 1997. 
(3) Is $|H|$ very ample?

The answer to (1) is usually given in two parts: the Riemann-Roch theorem computes $\chi(S, H)$, and then we need estimates for $h^{i}(S, H), i>0$. In particular, we may ask the following question related to (1):

$\left(1^{\prime}\right)$ When are $h^{1}(S, H)$ and $h^{2}(S, H)$ equal to zero?

One classical answer to $\left(1^{\prime}\right)$ is provided by Kodaira's vanishing theorem: if $L$ is any ample line bundle on $S$, then $h^{i}(S,-L)=0$ for all $i<2$; therefore, by Serre duality, we have $h^{i}\left(S, K_{S}+L\right)=0$ for all $i>0$. To answer $\left(1^{\prime}\right.$ ), write $H=K_{S}+L$ (thus defining $L$ as $H-K_{S}$ ); if $L$ is ample, then $h^{i}(S, H)=0$ for all $i>0$.

For questions (2) and (3), Reider [Rei] gave an answer which again considers $H$ in the form of an adjoint line bundle, $H=K_{S}+L$ :

Proposition (cf. [Rei, Theorem 1]). If $L$ is a line bundle on $S$, $L^{2} \geq 5$ and $L \cdot C \geq 2$ for every curve $C \subset S$, then $\left|K_{S}+L\right|$ is base-pointfree. If $L^{2} \geq 10$ and $L \cdot C \geq 3$ for every curve $C$, then $\left|K_{S}+L\right|$ is very ample.

We note here that Kodaira's theorem holds in all dimensions. Reider's criterion was tentatively extended in higher dimensions in the form of Fujita's conjecture ([Fuj]): if $X$ is a smooth projective variety of dimension $n$, and $L$ is an ample line bundle on $X$, then $\left|K_{X}+m L\right|$ is base-point-free for $m \geq n+1$ and very ample for $m \geq n+2$. Fujita's conjecture for basepoint-freeness was proved in dimension 3 by Ein and Lazarsfeld ([EL]) and in dimension 4 by Kawamata ([Kaw]); more precise statements, which resemble Reider's criterion more closely, were also obtained. Very ampleness, however, is still open, even in dimension 3.

Kodaira's vanishing theorem and Reider's criterion are already very useful as stated. However, the applicability of Kodaira's theorem was greatly extended, first on surfaces, by Mumford, Ramanujam, Miyaoka, and then in all dimensions by Kawamata and Viehweg, as follows. First, the ampleness condition for $L$ can be relaxed to $L \cdot C \geq 0$ for every curve $C$ and $L^{2}>0$ ( $L$ nef and big). Second, and most important, assume that $L$ itself is not nef and big, but there is a nef and big $\mathbb{Q}$-divisor $M$ on $S$ $(M \in \operatorname{Div}(S) \otimes \mathbb{Q})$ such that $L=\lceil M\rceil$ (i.e. $L-M$ is an effective $\mathbb{Q}$-divisor $B$ whose coefficients are all $<1)$. Then we have $h^{i}\left(S, K_{S}+L\right)=0$ for all 
$i>0$, just as in Kodaira's theorem. ( $\mathbb{Q}$-divisors were first considered in this context in connection with the Zariski decomposition of effective divisors.)

In dimension $\geq 3$, the Kawamata-Viehweg vanishing theorem requires an extra hypothesis (the irreducible components of $\operatorname{Supp}(B)$ must cross normally); however, Sakai remarked that for surfaces this extra hypothesis is not necessary (see Proposition 1.2.1 in $\S 1$ ).

For base-point-freeness (question (2) above), Ein and Lazarsfeld ([EL]) proved a similar extension of Reider's criterion, expressing $H$ as $K_{S}+\lceil M\rceil$ for a $\mathbb{Q}$-divisor $M$ on $S$; if $M^{2}>4$ and $M \cdot C \geq 2$ for every curve $C$, then $|H|$ is base-point-free. They used this result in their proof of Fujita's conjecture for base-point-freeness in dimension 3. (In fact they used a more precise local version, involving the local multiplicities of $B=L-M$; see $\S 1$ below).

In this paper we give criteria for very ampleness of linear systems of the form $\left|K_{S}+B+M\right|, B=\lceil M\rceil-M$, as above. In particular, we prove the following result:

Theorem 1. Let $S, B$ and $M$ be as above, and assume that

(0.1) $M^{2}>2\left(\beta_{2}\right)^{2}$,

(0.2) $M \cdot C \geq 2 \beta_{1}$ for every irreducible curve $C \subset S$,

where $\beta_{2}, \beta_{1}$ are positive numbers satisfying the following inequalities:

(0.3) $\beta_{2} \geq 2$,

(0.4) $\beta_{1} \geq \frac{\beta_{2}}{\beta_{2}-1}$.

Then $\left|K_{S}+B+M\right|$ is very ample.

An immediate consequence of Theorem 1 is the following:

Corollary 2. Assume that $(S, B)$ is as before, and $M$ is an ample $\mathbb{Q}$ divisor on $S$ such that $B=\lceil M\rceil-M, M^{2}>(2+\sqrt{2})^{2}$, and $M \cdot C>2+\sqrt{2}$ for every curve $C \subset S$. Then $\left|K_{S}+B+M\right|$ is very ample. In particular, if $A$ is an ample divisor (with integer coefficients) on $S$, then $\left|K_{S}+\lceil a A\rceil\right|$ is very ample for every $a \in \mathbb{Q}, a>2+\sqrt{2}$.

Note that Reider's criterion implies only that $\left|K_{S}+a A\right|$ is very ample for every integer $a \geq 4$. 
As in [EL, §2] (where the analogue for base-point-freeness was proved), we prove a local version of Theorem 1 , with the numerical conditions on $M$ relaxed in terms of local multiplicities of $B$.

As we mentioned earlier, the result for base-point-freeness on surfaces with boundary (i.e. for $\mathbb{Q}$-divisors $M$ ) was used in [EL] in the proof of Fujita's Conjecture in dimension 3. Similarly, we expect that the proof of the analogous result for very ampleness in dimension 3 will use very ampleness for $\mathbb{Q}$-divisors on surfaces. However, a natural and interesting question is whether or not the results for $\mathbb{Q}$-divisors on surfaces have any useful applications to the study of linear systems on surfaces. An example we discuss in $\S 4$ seems to indicate an affirmative answer. While the results proved in $\S 4$ can be obtained with other methods, our example shows how our $\mathbb{Q}$-Reider theorem extends the applicability of Reider's original result in the same way the Kawamata-Viehweg vanishing theorem extends the range of applicability of Kodaira's vanishing theorem. The usefulness of considering local multiplicities of $B$ is also evident in this example.

The paper is divided as follows: $\S 1$ is devoted to base-point-freeness. The results discussed in this section, with one exception, were proved in [EL]; I include a (slightly modified) proof to fix the ideas and notations for

the later sections. As one might expect, separation of points is relatively easy (at least in principle); it is discussed in $\S 2$. Then we move on to separation of tangent directions in $\S 3$. This part is surprisingly delicate; in particular the "multiplier ideal" method of Ein-Lazarsfeld, or Kawamata's equivalent "log-canonical threshold" formalism, do not work in this context. We explain the geometric contents of our method in the beginning of $\S 3$. Theorem 1 follows from Proposition 4 in $\S 2$ and Proposition 5 in $\S 3$. Finally, $\S 4$ contains the example mentioned earlier.

The author is grateful to L. Ein, R. Lazarsfeld, S. Lee, and N. Mohan Kumar for their many useful suggestions.

\section{Base-point-freeness}

(1.1) Let $S$ be a smooth projective surface over $\mathbb{C}$, and $B=\sum b_{i} C_{i}$ a fixed effective $\mathbb{Q}$-divisor on $S$ with $0 \leq b_{i}<1$ for all $i$. (The pair $(S, B)$ is sometimes called a "surface with boundary", whence the title of this paper.) Let $M$ be a $\mathbb{Q}$-divisor on $S$ such that $B+M$ has integer coefficients. 
We assume throughout this paper that $M$ is nef and big, i.e. that $M \cdot C \geq 0$ for every curve $C \subset S$ and $M^{2}>0$.

(1.2) For convenience, we gather here two technical results which we use time and again in our proofs.

(1.2.1) We use the following variants of the Kawamata-Viehweg vanishing theorem, which hold on smooth surfaces:

Theorem. (a) (cf. [EL, Lemma 1.1]) Let $S$ be a smooth projective surface over $\mathbb{C}$, and let $M$ be a nef and big $\mathbb{Q}$-divisor on $S$. Then

$$
H^{i}\left(S, K_{S}+\lceil M\rceil\right)=0, \quad \forall i>0 .
$$

(b) (cf. [EL, Lemma 2.4]) Assume moreover that $C_{1}, \ldots, C_{k}$ are distinct irreducible curves on $S$ which have integer coefficients in $M$. Assume that $M \cdot C_{j}>0$ for all $j=1, \ldots, k$. Then

$$
H^{i}\left(S, K_{S}+\lceil M\rceil+C_{1}+\cdots+C_{k}\right)=0, \quad \forall i>0 .
$$

(1.2.2) We use the following criterion for base-point-freeness, respectively very ampleness, on a complete Gorenstein curve (cf. [Har2]):

Proposition. Let $D$ be a Cartier divisor on the integral projective Gorenstein curve $C$. Then:

(a) $\operatorname{deg}(D) \geq 2 \Longrightarrow$ the complete linear system $\left|K_{C}+D\right|$ is base-pointfree;

(b) $\operatorname{deg}(D) \geq 3 \Longrightarrow\left|K_{C}+D\right|$ is very ample.

Proof. See $[$ Har2, §1] for the relevant definitions (generalized divisors on $C$, including 0 -dimensional subschemes; degree; etc.)

We prove (b); the proof of (a) is similar. By [Har2, Proposition 1.5], it suffices to show that $h^{0}\left(C, K_{C}+D-Z\right)=h^{0}\left(C, K_{C}+D\right)-2$ for every 0-dimensional subscheme $Z \subset C$ of length 2. Consider the exact sequence:

$$
0 \longrightarrow \mathcal{O}_{C}\left(K_{C}+D-Z\right) \longrightarrow \mathcal{O}_{C}\left(K_{C}+D\right) \longrightarrow \mathcal{O}_{C}\left(K_{C}+D\right) \otimes \mathcal{O}_{Z} \longrightarrow 0
$$

As $\mathcal{O}_{C}\left(K_{C}+D\right) \otimes \mathcal{O}_{Z} \cong \mathcal{O}_{Z}$ has length 2 , the conclusion will follow from the vanishing of $H^{1}\left(C, K_{C}+D-Z\right)$. By Serre duality (cf. [Har2, Theorem 1.4]), $H^{1}\left(C, K_{C}+D-Z\right) \cong H^{0}(C, Z-D)$, and $H^{0}(C, Z-D)=0$ due to $\operatorname{deg}(Z-D)=2-\operatorname{deg}(D)<0$. 
(1.3) Fix a point $p \in S$. In this section we give sufficient conditions for $\left|K_{S}+B+M\right|$ to be free at $p$.

(1.3.1) Notation. $\quad \mu=\operatorname{ord}_{p}(B) \stackrel{\text { def }}{=} \sum b_{i} \cdot \operatorname{mult}_{p}\left(C_{i}\right) \quad\left(B=\sum b_{i} C_{i}\right)$.

Proposition 3. $\left|K_{S}+B+M\right|$ is free at $p$ in each of the following cases:

1. $\mu \geq 2$;

2. $0 \leq \mu<2 ; M^{2}>\left(\beta_{2}\right)^{2}, M \cdot C \geq \beta_{1}$ for every irreducible curve $C \subset S$ such that $p \in C$, where $\beta_{2}, \beta_{1}$ are positive numbers which satisfy the inequalities:

$$
\begin{aligned}
& \beta_{2} \geq 2-\mu, \\
& \beta_{1} \geq \min \left\{(2-\mu) ; \frac{\beta_{2}}{\beta_{2}-(1-\mu)}\right\} .
\end{aligned}
$$

Remark. Explicitly, the minimum in (1.3.3) is given by:

$$
\min \left\{(2-\mu) ; \frac{\beta_{2}}{\beta_{2}-(1-\mu)}\right\}=\left\{\begin{array}{cc}
2-\mu & \text { if } 1 \leq \mu<2 \\
\frac{\beta_{2}}{\beta_{2}-(1-\mu)} & \text { if } 0 \leq \mu<1 .
\end{array}\right.
$$

In other words, when $0 \leq \mu<2$, the inequalities $\beta_{2} \geq 2-\mu$ and $\beta_{1} \geq 2-\mu$ suffice. When $\mu<1$ the inequality for $\beta_{1}$ can be relaxed to

$$
\beta_{1} \geq \frac{\beta_{2}}{\beta_{2}-(1-\mu)}
$$

this last part (which is useful in applications, cf. §4) is not contained in [EL].

\section{Proof of Proposition 3}

(1.4) Let $f: S_{1} \rightarrow S$ be the blowing-up of $S$ at $p$, and let $E \subset S_{1}$ be the exceptional divisor of $f$. We have $f^{*} B=f^{-1} B+\mu E ;\left\lfloor f^{-1} B\right\rfloor=0$, and therefore

$$
\begin{aligned}
K_{S_{1}}+\left\lceil f^{*} M\right\rceil & =f^{*} K_{S}+E+\left\lceil f^{*}(B+M)-f^{*} B\right\rceil \\
& =f^{*}\left(K_{S}+B+M\right)+E-\left\lfloor f^{*} B\right\rfloor \\
& =f^{*}\left(K_{S}+B+M\right)-(\lfloor\mu\rfloor-1) E .
\end{aligned}
$$


(1.5) case $t=\lfloor\mu\rfloor-1$ is a positive integer; since $f^{*} M$ is nef and big on $S_{1}$, the vanishing theorem $(1.2 .1)$ (a) yields

$$
H^{1}\left(S_{1}, K_{S_{1}}+\left\lceil f^{*} M\right\rceil\right)=0,
$$

and therefore (using (1.4.1) and the projection formula)

$$
H^{1}\left(S, \mathcal{O}_{S}\left(K_{S}+B+M\right) \otimes \mathfrak{m}_{p}{ }^{t}\right)=0
$$

where $\mathfrak{m}_{p}$ is the maximal ideal of $\mathcal{O}_{S}$ at $p$. The conclusion follows from the surjectivity of the restriction map

$$
H^{0}\left(S, K_{S}+B+M\right) \longrightarrow H^{0}\left(S, \mathcal{O}_{S}\left(K_{S}+B+M\right) \otimes \mathcal{O}_{S} / \mathfrak{m}_{p}{ }^{t}\right) \cong \mathcal{O}_{S} / \mathfrak{m}_{p}{ }^{t}
$$

(1.5.3) Remark. In fact we proved that $\left|K_{S}+B+M\right|$ separates $s$-jets at $p$, if $\mu \stackrel{\text { def }}{=} \operatorname{ord}_{p}(B) \geq s+2$.

(1.6) Now assume that $\mu<2$, and $M^{2}>\left(\beta_{2}\right)^{2}$ with $\beta_{2} \geq 2-\mu$, etc.

(1.6.1) Claim. We can find an effective $\mathbb{Q}$-divisor $D$ on $S$ such that $\operatorname{ord}_{p}(D)=2-\mu$ and $D \underset{\mathbb{Q}}{\sim} t M$ for some $t \in \mathbb{Q}, 0<t<\frac{2-\mu}{\beta_{2}}$. ( $\underset{\mathbb{Q}}{ }$ denotes $\mathbb{Q}$-linear equivalence, i.e. $m D$ and $m t M$ have integer coefficients and are linearly equivalent for some suitably large and divisible integer $m$.)

Proof of (1.6.1). By Riemann-Roch, $\operatorname{dim}|n M|$ grows like $\frac{M^{2}}{2} n^{2}>$ $\frac{\left(\beta_{2}\right)^{2}}{2} n^{2}$ for $n$ sufficiently large and divisible (such that $n M$ has integer coefficients). Since $\operatorname{dim}\left(\mathcal{O}_{S, p} / \mathfrak{m}_{p}{ }^{n}\right)$ grows like $\frac{n^{2}}{2}$, for suitable $n$ we can find $G \in|n M|$ with $\operatorname{ord}_{p}(G)>\beta_{2} n$.

Take $D=r G, r=\frac{2-\mu}{\operatorname{ord}_{p}(G)}$; then $\operatorname{ord}_{p}(D)=2-\mu$, and $D \underset{\mathbb{Q}}{\sim} t M$ for $t=r n<\frac{2-\mu}{\beta_{2} n} n=\frac{2-\mu}{\beta_{2}}$.

Note that $\frac{2-\mu}{\beta_{2}} \leq 1$, by $(1.3 .2)$, so that $t<1$; therefore $M-D \underset{\mathbb{Q}}{\sim}(1-t) M$ is still nef and big.

(1.7) Recall that $B=\sum b_{i} C_{i}$, for distinct irreducible curves $C_{i} \subset S$. Write $D=\sum d_{i} C_{i}$ (we allow some coefficients $b_{i}$ and $d_{i}$ to be zero); $d_{i} \in \mathbb{Q}$, $d_{i} \geq 0$, and $\operatorname{ord}_{p}(D)=\sum d_{i} \cdot \operatorname{mult}_{p}\left(C_{i}\right)=2-\mu$.

Let $D_{i}=f^{-1} C_{i} \subset S_{1}$ be the strict transform of $C_{i}$; then $f^{*} B=$ $\sum b_{i} D_{i}+\mu E, f^{*} D=\sum d_{i} D_{i}+(2-\mu) E, K_{S_{1}}=f^{*} K_{S}+E$, and

$$
K_{S_{1}}-f^{*}\left(K_{S}+B+D\right)=-E-\sum\left(b_{i}+d_{i}\right) D_{i} .
$$


Definition. $(S, B, D)$ is partially log-canonical at $p(P L C$ at $p)$ if $-\left(b_{i}+d_{i}\right) \geq-1$ (i.e. $\left.b_{i}+d_{i} \leq 1\right)$ for every $i$ such that $p \in C_{i}$. (The general definition requires the coefficient of $E$ to be $\geq-1$, too; in our case that coefficient is equal to -1 .)

Note that $P L C$ is not the same as log-canonical (cf. [KMM, Definition $0-2-10]$ ), because $f$ is not an embedded resolution of $(S, B+D)$.

(1.8) If $(S, B, D)$ is $P L C$ at $p$, then the proof is almost as simple as in the case $\mu \geq 2$ :

$$
\begin{aligned}
K_{S_{1}} & +\left\lceil f^{*}(M-D)\right\rceil \\
& =f^{*} K_{S}+E+f^{*}(B+M)-\left\lfloor f^{*}(B+D)\right\rfloor \\
& =f^{*}\left(K_{S}+B+M\right)+E-2 E-\sum\left\lfloor b_{i}+d_{i}\right\rfloor D_{i} \\
& =f^{*}\left(K_{S}+B+M\right)-E-\sum^{\prime} D_{i}-N_{1},
\end{aligned}
$$

where $\sum^{\prime} D_{i}$ extends over those $i$ for which $p \in C_{i}$ and $b_{i}+d_{i}=1$ (if any), and $N_{1}$ is an effective divisor supported away from $E$.

$f^{*} M \cdot D_{i}=M \cdot C_{i}>0$ if $p \in C_{i}$; therefore (1.2.1)(b) yields:

$$
H^{1}\left(S_{1}, f^{*}\left(K_{S}+B+M\right)-E-N_{1}\right)=0 .
$$

Arguing as in (1.5), we can show that $p \notin \mathrm{Bs}\left|K_{S}+B+M-N\right|$, where $N=f_{*} N_{1}$; i.e., $\exists \Lambda \in\left|K_{S}+B+M-N\right|$ with $p \notin \operatorname{Supp}(\Lambda)$. Then $\Lambda+N \in\left|K_{S}+B+M\right|$ and $p \notin \operatorname{Supp}(\Lambda+N)$, as required.

Note that we haven't used (1.3.3) yet; all we needed so far was $\beta_{1}>0$.

(1.9) Finally, assume that $(S, B, D)$ is not $P L C$ at $p$. Then $b_{j}+d_{j}>1$ for some $j$ with $p \in C_{j}$. In fact, since $2=\operatorname{ord}_{p}(B+D)=\sum\left(b_{i}+d_{i}\right)$. $\operatorname{mult}_{p}\left(C_{i}\right)$, there can be at most one $C_{j}$ through $p$ with $b_{j}+d_{j}>1$, and then that $C_{j}$ must be smooth at $p$ and also $b_{i}+d_{i}<1$ for all $i \neq j$ with $p \in C_{i}$. Let that $j$ be 0 ; thus $b_{0}+d_{0}>1, C_{0}$ is smooth at $p$, and $b_{i}+d_{i}<1$ if $i \neq 0$ and $p \in C_{i}$. We say that $C_{0}$ is the critical curve at $p$.

Let $c$ be the $P L C$ threshold of $(S, B, D)$ at $p$ :

$$
c=\max \left\{\lambda \in \mathbb{Q}_{+} \mid(S, B, \lambda D) \text { is } P L C \text { at } p\right\} ;
$$

explicitly, $b_{0}+c d_{0}=1$, i.e. $c=\frac{1-b_{0}}{d_{0}}$. Note that $0<c<1$. 
$M-c D \underset{\mathbb{Q}}{\sim}(1-c t) M$ is still nef and big on $S$, and we have:

$$
\begin{aligned}
K_{S}+\lceil M-c D\rceil & =K_{S}+B+M-\lfloor B+c D\rfloor \\
& =K_{S}+B+M-C_{0}-N,
\end{aligned}
$$

with $p \notin \operatorname{Supp}(N)$. (If $p \in C_{i}$ and $i \neq 0$ then $b_{i}+d_{i}<1$, and therefore $b_{i}+c d_{i}<1$, too, because $c<1$; hence $p \notin \operatorname{Supp}(N)$.)

(1.2.1)(a) yields $H^{1}\left(S, K_{S}+B+M-C_{0}-N\right)=0$, and therefore the restriction map $H^{0}\left(S, K_{S}+B+M-N\right) \rightarrow H^{0}\left(C_{0},\left.\left(K_{S}+B+M-N\right)\right|_{C_{0}}\right)$ is surjective. Hence it suffices to show that $p \notin \mathrm{Bs}\left|\left(K_{S}+B+M-N\right)\right|_{C_{0}} \mid$.

We have

$$
K_{S}+B+M-N=K_{S}+\lceil M-c D\rceil+C_{0},
$$

and therefore $\left.\left(K_{S}+B+M-N\right)\right|_{C_{0}}=K_{C_{0}}+\left.\lceil M-c D\rceil\right|_{C_{0}}$; by (1.2.2)(a), it suffices to show that $\lceil M-c D\rceil \cdot C_{0} \geq 2$. In any event $\lceil M-c D\rceil \cdot C_{0}$ is an integer; we will show that $\lceil M-c D\rceil \cdot C_{0}>1$.

$\lceil M-c D\rceil=(M-c D)+\Delta$, where $\Delta=\lceil M-c D\rceil-(M-c D)=$ $\lceil(M+B)-(B+c D)\rceil-(M-c D)=(M+B)-\lfloor B+c D\rfloor-(M-c D)=$ $(B+c D)-\lfloor B+c D\rfloor=\{B+c D\} . \Delta$ is an effective divisor which intersects $C_{0}$ properly, because $C_{0}$ has integer coefficient (namely, 1) in $B+c D$. Moreover, in a neighborhood of $p$ we have $\{B+c D\}=(B+c D)-C_{0}$, because $B+c D=C_{0}+\sum_{i \neq 0}\left(b_{i}+c d_{i}\right) C_{i}$, and $0 \leq b_{i}+c d_{i}<1$ for every $i \neq 0$ such that $p \in C_{i}$. In particular, we have

$$
\operatorname{ord}_{p}(\Delta)=\operatorname{ord}_{p}(B+c D)-1=\mu+c(2-\mu)-1 \text {. }
$$

$$
\begin{aligned}
& \lceil M-c D\rceil \cdot C_{0} \\
& =(M-c D) \cdot C_{0}+\Delta \cdot C_{0} \geq(1-c t) M \cdot C_{0}+\operatorname{ord}_{p}(\Delta) \\
& \geq(1-c t) \beta_{1}+\mu+c(2-\mu)-1 .
\end{aligned}
$$

Therefore the inequality $\lceil M-c D\rceil \cdot C_{0}>1$ follows from

$$
(1-c t) \beta_{1}>(1-c)(2-\mu) .
$$

If $\beta_{1} \geq 2-\mu$ then (1.10.1) is trivial, because $t<1 \Longrightarrow 1-c t>1-c$.

(1.11) When $\mu<1$ the inequality we assume for $\beta_{1}$ (namely, (1.3.4)) is weaker than $\beta_{1} \geq 2-\mu$. However, in this case the equation $B+c D=$ 
$C_{0}+$ other terms yields a nontrivial lower bound for $c$ : $\mu+c(2-\mu)=$ $\operatorname{ord}_{p}(B+c D) \geq \operatorname{ord}_{p}\left(C_{0}\right)=1$, and therefore $c \geq \frac{1-\mu}{2-\mu}>0$.

The inequality (1.10.1) can also be written as

$$
c\left(2-\mu-t \beta_{1}\right)>2-\mu-\beta_{1} .
$$

We may assume that $\beta_{1}<2-\mu$ (or else (1.10.1) is already proved). We have $c \geq \frac{1-\mu}{2-\mu}, t<\frac{2-\mu}{\beta_{2}}$ (see (1.6.1)), and $\frac{1-\mu}{\beta_{2}} \leq 1-\frac{1}{\beta_{1}}$ (by (1.3.4)); therefore

$$
\begin{aligned}
c\left(2-\mu-t \beta_{1}\right) & >\frac{1-\mu}{2-\mu}\left(2-\mu-\frac{2-\mu}{\beta_{2}} \beta_{1}\right)=\left(1-\mu-\frac{1-\mu}{\beta_{2}} \beta_{1}\right) \\
& \geq(1-\mu)-\left(1-\frac{1}{\beta_{1}}\right) \beta_{1}=2-\mu-\beta_{1} .
\end{aligned}
$$

(1.11.1) is proved. This concludes the proof of proposition 3 .

\section{Separation of points}

(2.1) Let $(S, B, M)$ be as in (1.1). Fix two distinct points $p, q \in S$. In this section we give criteria for $\left|K_{S}+B+M\right|$ to separate $(p, q)$.

Note that in each case $\left|K_{S}+B+M\right|$ is free at $p$ and $q$, by proposition 3, and therefore it suffices to find $s \in H^{0}\left(S, K_{S}+B+M\right)$ such that $s(p)=$ $0, s(q) \neq 0$, or vice-versa.

Notation. $\mu_{p}=\operatorname{ord}_{p}(B), \quad \mu_{q}=\operatorname{ord}_{q}(B)$.

Proposition 4. $\left|K_{S}+B+M\right|$ separates $(p, q)$ in each of the following cases:

1. $\mu_{p} \geq 2$ and $\mu_{q} \geq 2$;

2. $\mu_{q} \geq 2 ; 0 \leq \mu_{p}<2 ; M^{2}>\left(\beta_{2}\right)^{2}, M \cdot C \geq \beta_{1}$ for every irreducible curve $C \subset S$ passing through $p$, where $\beta_{2}, \beta_{1}$ are positive numbers which satisfy (1.3.2) and (1.3.3) for $\mu=\mu_{p}$;

3. $0 \leq \mu_{p}<2$ and $0 \leq \mu_{q}<2 ; M^{2}>\left(\beta_{2, p}\right)^{2}+\left(\beta_{2, q}\right)^{2}$, and

(i) $M \cdot C \geq \beta_{1, p}$ for every curve $C \subset S$ passing through $p$,

(ii) $M \cdot C \geq \beta_{1, q}$ for every curve $C \subset S$ passing through $q$,

(iii) $M \cdot C \geq \beta_{1, p}+\beta_{1, q}$ if $C$ passes through both $p$ and $q$, 
where $\beta_{2, p}, \beta_{1, p} ; \beta_{2, q}, \beta_{1, q}$ are positive numbers which satisfy the inequalities

$$
\begin{aligned}
& \beta_{2, p} \geq 2-\mu_{p}, \quad \beta_{2, q} \geq 2-\mu_{q} \\
& \beta_{1, p} \geq \min \left\{\left(2-\mu_{p}\right) ; \frac{\beta_{2, p}}{\beta_{2, p}-\left(1-\mu_{p}\right)}\right\}, \text { and similarly for } \beta_{1, q} .
\end{aligned}
$$

\section{Proof of Proposition 4}

(2.2) Let $f: S_{1} \rightarrow S$ be the blowing-up of $S$ at $p$ and $q$, with exceptional curves $E_{p}, E_{q}$. As in (1.4), we have:

$$
K_{S_{1}}+\left\lceil f^{*} M\right\rceil=f^{*}\left(K_{S}+B+M\right)-\left(\left\lfloor\mu_{p}\right\rfloor-1\right) E_{p}-\left(\left\lfloor\mu_{q}\right\rfloor-1\right) E_{q} .
$$

In particular, if $\mu_{p} \geq 2$ and $\mu_{q} \geq 2$ (case 1 of the proposition), we get

$$
H^{1}\left(S, \mathcal{O}_{S}\left(K_{S}+B+M\right) \otimes \mathfrak{m}_{p}{ }^{t_{p}} \otimes \mathfrak{m}_{q}{ }^{t_{q}}\right)=0
$$

for positive integers $t_{p}, t_{q}$ (compare to (1.5.2)); the conclusion follows as in (1.5).

(2.3) Next assume that $\mu_{p}<2, \mu_{q} \geq 2, M^{2}>\left(\beta_{2}\right)^{2}$ with $\beta_{2} \geq 2-\mu_{p}$, etc. (case 2 of the proposition). Write $\mu=\mu_{p}$. As in (1.6.1), we can find an effective $\mathbb{Q}$-divisor $D$ on $S$ such that $\operatorname{ord}_{p}(D)=2-\mu$ and $D \underset{\mathbb{Q}}{\sim} t M$ for some $t \in \mathbb{Q}, 0<t<\frac{2-\mu}{\beta_{2}}$.

If $(S, B, D)$ is $P L C$ at $p$, the argument of (1.8) yields a vanishing

$$
H^{1}\left(S_{1}, f^{*}\left(K_{S}+B+M\right)-E_{p}-N_{0}\right)=0
$$

where $N_{0}$ is an effective divisor supported away from $E_{p}$. Note that in this case $N_{0} \geq E_{q}$, because $\mu_{q} \geq 2$. Indeed, (2.3.1) is obtained by applying (1.2.1)(b) to

$$
\begin{aligned}
& K_{S_{1}}+\left\lceil f^{*}(M-D)\right\rceil \\
& \quad=f^{*}\left(K_{S}+B+M\right)-E_{p}-t_{q} E_{q}-\sum\left\lfloor b_{i}+d_{i}\right\rfloor D_{i} \\
& \quad=f^{*}\left(K_{S}+B+M\right)-E_{p}-t_{q} E_{q}-\sum^{\prime} D_{i}-N_{1},
\end{aligned}
$$

where $\sum^{\prime} D_{i}$ and $N_{1}$ are as in (1.8.1) and $t_{q}=\left\lfloor\mu_{q}+\operatorname{ord}_{q}(D)\right\rfloor-1$ is an integer, $t_{q} \geq 1$; then $N_{0}=N_{1}+t_{q} E_{q} \geq E_{q}$. 
The vanishing (2.3.1) implies the surjectivity of the restriction map

$$
\begin{aligned}
H^{0}\left(S_{1}, f^{*}\left(K_{S}+B\right.\right. & \left.+M)-N_{0}\right) \\
& \longrightarrow H_{0}\left(E_{p},\left.\left(f^{*}\left(K_{S}+B+M\right)-N_{0}\right)\right|_{E_{p}}\right) \cong \mathbb{C}
\end{aligned}
$$

(note that $\left.f^{*}\left(K_{S}+B+M\right)\right|_{E_{p}}$ is trivial, and so is $\left.N_{0}\right|_{E_{p}}$ because $N_{0} \cap E_{p}=\emptyset$ ).

Hence we can find $\Gamma \in\left|f^{*}\left(K_{S}+B+M\right)-N_{0}\right|$ such that $\Gamma \cap E_{p}=\emptyset$. As $\Gamma+N_{0} \in\left|f^{*}\left(K_{S}+B+M\right)\right|$, we have $\Gamma+N_{0}=f^{*} \Lambda$ for some $\Lambda \in\left|K_{S}+B+M\right|$. Moreover, $p \notin \operatorname{Supp}(\Lambda)$, because $f^{*} \Lambda \cap E_{p}=\emptyset$, but $q \in \operatorname{Supp}(\Lambda)$, because $f^{*} \Lambda=\Gamma+N_{0} \geq E_{q}$. Thus $\left|K_{S}+B+M\right|$ separates $(p, q)$ in this case.

(2.4) Now assume that $(S, B, D)$ is not $P L C$ at $p$. Let $c, C_{0}$ be the $P L C$ threshold and the critical curve at $p$, as in $\S 1,(1.9)-(1.11)$. Let $\phi: S_{2} \longrightarrow S$ be the blowing-up of $S$ at $q$ (only), with exceptional curve $F_{q}$. Let $C_{0}^{\prime} \subset S_{2}$ be the proper transform of $C_{0}$ in $S_{2}$. Let $p^{\prime}=\phi^{-1}(p)$. We have:

$$
K_{S_{2}}+\left\lceil\phi^{*}(M-c D)\right\rceil=\phi^{*}\left(K_{S}+B+M\right)-C_{0}^{\prime}-N_{0},
$$

where $p^{\prime} \notin \operatorname{Supp}\left(N_{0}\right)$, as in (1.9), and $N_{0} \geq F_{q}$, as in (2.3).

The argument in (1.9)-(1.11) shows that there exists $\Gamma \in \mid \phi^{*}\left(K_{S}+B+\right.$ $M)-N_{0} \mid$ with $p^{\prime} \notin \operatorname{Supp}(\Gamma)$. Now the proof can be completed as in the last part of (2.3).

(2.5) Finally, consider the case $\mu_{p}<2$ and $\mu_{q}<2$, with $M^{2}>\left(\beta_{2, p}\right)^{2}+$ $\left(\beta_{2, q}\right)^{2}$, etc. (case 3 of the proposition).

As in (1.6.1), we can find $G \in|n M|$ with $\operatorname{ord}_{p}(G)>\beta_{2, p} n$ and $\operatorname{ord}_{q}(G)$ $>\beta_{2, q} n$. Let $r=\max \left\{\frac{2-\mu_{p}}{\operatorname{ord}_{p}(G)}, \frac{2-\mu_{q}}{\operatorname{ord}_{q}(G)}\right\}$, and $D=r G$. Then $\operatorname{ord}_{p}(D) \geq$ $2-\mu_{p}$ and $\operatorname{ord}_{q}(G) \geq 2-\mu_{q}$, and at least one of the last two inequalities is an equality. Without loss of generality we may assume that $\operatorname{ord}_{p}(D)=2-\mu_{p}$ and $m_{q} \stackrel{\text { def }}{=} \operatorname{ord}_{q}(D) \geq 2-\mu_{q}$. We have $D \underset{\mathbb{Q}}{\sim} t M$, with

$$
0<t=r n=\frac{2-\mu_{p}}{\operatorname{ord}_{p}(G)} n<\frac{2-\mu_{p}}{\beta_{2, p}} \leq 1 ;
$$

also, $m_{q}=\operatorname{ord}_{q}(D)=r \cdot \operatorname{ord}_{q}(G)>r \cdot\left(\beta_{2, q} n\right)=t \beta_{2, q}$, and therefore

$$
t<\frac{m_{q}}{\beta_{2, q}}
$$

(this is the analogue of (2.5.1) at $q$ ). 
If $(S, B, D)$ is $P L C$ at $p$, then (1.2.1)(b) yields

$$
H^{1}\left(S_{1}, f^{*}\left(K_{S}+B+M\right)-E_{p}-N_{0}\right)=0,
$$

with $N_{0} \cap E_{p}=\emptyset, N_{0} \geq E_{q}$ (the computation in (2.3.2) applies unchanged in this situation). In this case we conclude as in (2.3).

(2.6) Now assume that $(S, B, D)$ is not $P L C$ at $p$. Let $c, C_{0}$ be the $P L C$ threshold and the critical curve at $p$. (1.2.1)(a) yields

$$
H^{1}\left(S_{1}, f^{*}\left(K_{S}+B+M\right)-D_{0}-N_{0}\right)=0, \quad N_{0} \cap E_{p}=\emptyset .
$$

If $N_{0} \cap E_{q} \neq \emptyset$, we use (2.6.1) to find $\Gamma \in\left|f^{*}\left(K_{S}+B+M\right)-N_{0}\right|$ which does not pass through $\tilde{p}=D_{0} \cap E_{p}$; the proof is the same as in (1.9)-(1.11). Then the conclusion follows as in (2.3).

Assume that $N_{0} \cap E_{q}=\emptyset$. We discuss separately the subcases $q \in C_{0}$ and $q \notin C_{0}$. If $q \in C_{0}$, we separate $(p, q)$ on $C_{0}$. If $q \notin C_{0}$, we reverse the roles of $p$ and $q$.

(2.7) First consider the subcase $q \in C_{0}$. The vanishing (2.6.1) implies

$$
H^{1}\left(S, K_{S}+B+M-C_{0}-N\right)=0,
$$

with $N=f_{*} N_{0}, \operatorname{Supp}(N) \cap\{p, q\}=\emptyset$. Consequently, the restriction map

$$
H^{0}\left(S, K_{S}+B+M-N\right) \longrightarrow H^{0}\left(C_{0},\left.\left(K_{S}+B+M-N\right)\right|_{C_{0}}\right)
$$

is surjective, and it suffices to show that $\left|\left(K_{S}+B+M-N\right)\right|_{C_{0}} \mid$ separates $(p, q)$ on $C_{0}$. As in (1.9.1), we have

$$
\left.\left(K_{S}+B+M-N\right)\right|_{C_{0}}=K_{C_{0}}+\left.\lceil M-c D\rceil\right|_{C_{0}} ;
$$

by $(1.2 .2)(\mathrm{b})$ it is enough to show that $\lceil M-c D\rceil \cdot C_{0}>2$ (and consequently $\geq 3)$.

We proceed as in $\S 1$ : $\lceil M-c D\rceil=(M-c D)+\Delta$, with $\Delta=\{B+c D\}$; $\Delta$ and $C_{0}$ intersect properly, and $\operatorname{ord}_{p}(\Delta)=\mu_{p}+c\left(2-\mu_{p}\right)-1, \operatorname{ord}_{q}(\Delta)=$ $\mu_{q}+c m_{q}-1$ (note that $N_{0} \cap E_{q}=\emptyset \Longrightarrow$ the only component with coefficient $\geq 1$ of $B+c D$ through $q$ is $C_{0}$, and moreover $C_{0}$ must be smooth at $q$ ). Therefore

$$
\begin{aligned}
& \lceil M-c D\rceil \cdot C_{0} \\
= & (M-c D) \cdot C_{0}+\Delta \cdot C_{0} \\
\geq & (1-c t) M \cdot C_{0}+\operatorname{ord}_{p}(\Delta)+\operatorname{ord}_{q}(\Delta) \\
\geq & (1-c t)\left(\beta_{1, p}+\beta_{1, q}\right)+\left(\mu_{p}+c\left(2-\mu_{p}\right)-1\right)+\left(\mu_{q}+c m_{q}-1\right)
\end{aligned}
$$


$\left(M \cdot C_{0} \geq \beta_{1, p}+\beta_{1, q}\right.$, because this time $C_{0}$ passes through both $p$ and $\left.q.\right)$

Hence $\lceil M-c D\rceil \cdot C_{0}>2$ follows from

$$
(1-c t)\left(\beta_{1, p}+\beta_{1, q}\right)+\left(\mu_{p}+c\left(2-\mu_{p}\right)-1\right)+\left(\mu_{q}+c m_{q}-1\right)>2,
$$

which in turn follows from the following two inequalities:

$$
\begin{gathered}
(1-c t) \beta_{1, p}+\left(\mu_{p}+c\left(2-\mu_{p}\right)-1\right)>1 \text { and } \\
(1-c t) \beta_{1, q}+\left(\mu_{q}+c m_{q}-1\right)>1 .
\end{gathered}
$$

(2.7.3) is proved like (1.10.1) in $\S 1$ : if $\beta_{1, p} \geq 2-\mu_{p}$, then $t<1 \Longrightarrow$ $(1-c t) \beta_{1, p}>(1-c)\left(2-\mu_{p}\right) \Longrightarrow(2.7 .3)$. If $\beta_{1, p}<2-\mu_{p}$ (which can happen only if $\left.\mu_{p}<1\right)$, then we have $c \geq \frac{1-\mu_{p}}{2-\mu_{p}}$ as in (1.11), $t<\frac{2-\mu_{p}}{\beta_{2, p}}$ by (2.5.1), and $\frac{1-\mu_{p}}{\beta_{2, p}} \leq 1-\frac{1}{\beta_{1, p}}$ by (2.1.2), and therefore (2.7.3) follows as in (1.11).

(2.7.4) is proved similarly. First, since $m_{q}=\operatorname{ord}_{q}(D) \geq 2-\mu_{q}$, the inequality is true when $\beta_{1, q} \geq 2-\mu_{q}$, as in the proof of (2.7.3) above. When $\beta_{1, q}<2-\mu_{q}$ we must have $\mu_{q}<1$; then $B+c D \geq C_{0} \Longrightarrow \mu_{q}+c m_{q} \geq 1 \Longrightarrow$ $c \geq \frac{1-\mu_{q}}{m_{q}}, t<\frac{m_{q}}{\beta_{2, q}}$ by (2.5.2), and $\frac{1-\mu_{q}}{\beta_{2, q}} \leq 1-\frac{1}{\beta_{1, q}}$ by (2.1.2); consequently

$$
\begin{aligned}
c\left(m_{q}-t \beta_{1, q}\right) & >\frac{1-\mu_{q}}{m_{q}}\left(m_{q}-\frac{m_{q}}{\beta_{2, q}} \beta_{1, q}\right) \\
& =\left(1-\mu_{q}\right)-\frac{1-\mu_{q}}{\beta_{2, q}} \beta_{1, q} \geq 2-\mu_{q}-\beta_{1, q},
\end{aligned}
$$

which yields (2.7.4).

Thus (2.7.2) is proved; this concludes the proof when $q \in C_{0}$.

(2.8) To complete the proof of the proposition in case 3, consider the remaining subcase, $q \notin C_{0}$. In this subcase separation of $(p, q)$ is obtained by reversing the roles of $p$ and $q$. Namely, let $D^{\prime}=\alpha D$, for the positive rational number $\alpha$ such that $\operatorname{ord}_{q}\left(D^{\prime}\right)=2-\mu_{q}$; that is, $\alpha=\frac{2-\mu_{q}}{\operatorname{ord}_{q}(D)}=\frac{2-\mu_{q}}{m_{q}}$. Note that $D^{\prime} \underset{\mathbb{Q}}{\sim} t^{\prime} M$, where $t^{\prime}=\alpha t<\frac{2-\mu_{q}}{m_{q}} \cdot \frac{m_{q}}{\beta_{2, q}}$ (by (2.5.2)), i.e.

$$
0<t^{\prime}<\frac{2-\mu_{q}}{\beta_{2, q}} \leq 1
$$

Let $c^{\prime}$ be the $P L C$ threshold for $\left(S, B, D^{\prime}\right)$ at $q$; note that $c^{\prime} \alpha>c\left(c^{\prime} \alpha\right.$ is the $P L C$ threshold of $(S, B, D)$ at $q$, and therefore $c<c^{\prime} \alpha$ follows from $N_{0} \cap E_{q}=\emptyset$ in (2.6.1)). This, in turn, implies $B+c^{\prime} D^{\prime}=B+c^{\prime} \alpha D \geq C_{0}$. 
If $\left(S, B, D^{\prime}\right)$ is $P L C$ at $q$ (i.e. if $\left.c^{\prime}=1\right)$, then (1.2.1)(b) yields

$$
H^{1}\left(S_{1}, f^{*}\left(K_{S}+B+M\right)-E_{q}-N_{0}^{\prime}\right)=0, \quad N_{0}^{\prime} \cap E_{q}=\emptyset
$$

(Compare to (2.5.3)).

If $\left(S, B, D^{\prime}\right)$ is not $P L C$ at $q$ (i.e. if $c^{\prime}<1$ ), and $C_{0}^{\prime}$ is the critical curve at $q$, then (1.2.1.) (a) yields

$$
H^{1}\left(S_{1}, f^{*}\left(K_{S}+B+M\right)-D_{0}^{\prime}-N_{0}^{\prime}\right)=0, \quad N_{0}^{\prime} \cap E_{q}=\emptyset
$$

(Compare to (2.6.1), noting that now $p$ and $q$ are interchanged.)

In both cases, the arguments in (1.8) and, respectively, (1.9)-(1.11) show that there exists $\Lambda \in\left|K_{S}+B+M-N^{\prime}\right|$ with $q \notin \operatorname{Supp}(\Lambda)$, where $N^{\prime}=f_{*} N_{0}^{\prime}$ is an effective divisor with $q \notin \operatorname{Supp}\left(N^{\prime}\right)$. Now, however, $N^{\prime} \geq$ $C_{0}$ (because $B+c^{\prime} D^{\prime} \geq C_{0}$, as noted earlier, and $q \notin C_{0} \Longrightarrow C_{0}$ is not discarded even when the vanishing theorem is used in the form (1.2.1)(b)); thus $\Gamma+N^{\prime} \in\left|K_{S}+B+M\right|$ passes through $p$ but not through $q$.

This completes the proof of proposition 4 .

\section{Separation of tangent directions}

(3.1) Let $(S, B, M)$ be as in $\S 1$. Fix a point $p \in S$. In this section we give criteria for $\left|K_{S}+B+M\right|$ to separate directions at $p$.

The statements (and proofs) are somewhat similar to those in $\S 2$. The main difference is in the part of the proof corresponding to the discussion in (2.8). So far in our proofs we worked with $M-c D$, where $c$ was always the $P L C$ threshold at some point or another; this made the arguments relatively transparent. In (2.8), when we passed from $c=P L C$ threshold at $p$ to $c^{\prime} \alpha=P L C$ threshold at $q$, the relevant fact was that $q \notin C_{0}$, where $C_{0}$ was the critical curve at $p$, and therefore $C_{0}$ did not affect the local computations around $q$. In separating tangent directions, the analogue is a curve $C_{0}$ through $p$, such that $\vec{v} \notin T_{p}\left(C_{0}\right)$ for some fixed $\vec{v} \in T_{p}(S), \vec{v} \neq \overrightarrow{0}$. Then we will have to increase $c$ to some larger value $c^{\prime}$, but clearly in that case $\left(S, B, c^{\prime} D\right)$ will no longer be $P L C$ at $p$. While this complicates the computations, the geometric idea is still the same: find a divisor $\Gamma \in$ $\left|K_{S}+B+M-C_{0}-N\right|, p \notin \operatorname{Supp}(N)$, such that $\Gamma$ does not pass through $p$; then $\Gamma+C_{0}+N$ has only one component through $p$, namely, $C_{0}$, and $\vec{v} \notin T_{p}\left(C_{0}\right)$ - therefore $\Gamma+C_{0}+N$ passes through $p$ and is not tangent to $\vec{v}$, as required. 
Another technical problem, which did not arise before, is that in some cases the "minimizing" curve $C_{0}$ may be singular at $p$. (This possibility is directly related to the need, in some cases, to increase $c$ beyond the $P L C$ threshold at $p$.) In those cases we separate the tangent directions on $C_{0}$, using $(1.2 .2)(\mathrm{b})$ (note that $C_{0}$ singular at $p \Longrightarrow T_{p}(S)=T_{p}\left(C_{0}\right)$ ); the vanishing (1.2.1) is then used to lift from $C_{0}$ to $S$.

(3.2) Let $S$ be a smooth surface, as before; let $p$ denote a point on $S$, and fix $\vec{v} \in T_{p}(S), \vec{v} \neq \overrightarrow{0}$. Let $Z$ denote the zero-dimensional subscheme of length 2 of $S$, corresponding to $(p, \vec{v})$; in local coordinates $(x, y)$ at $p$ such that $\vec{v}$ is tangent to $(y=0), Z$ is defined by the ideal $\mathcal{I}_{Z}=\left(x^{2}, y\right) \cdot \mathcal{O}_{S}$.

Let $f: S_{1} \rightarrow S$ be the blowing-up of $S$ at $p$, with exceptional curve $E_{p}$, and let $V \in E_{p}$ correspond to (the direction of) $\vec{v}$. Let $g: S_{2} \rightarrow S_{1}$ be the blowing-up of $S_{1}$ at $V$, with exceptional curve $F_{\vec{v}}$, and let $F_{p}=g^{-1} E_{p}$. Let $h=f \circ g$. Write

$$
h^{*} B=h^{-1} B+\mu_{p} F_{p}+\mu_{\vec{v}} F_{\vec{v}}
$$

$\mu_{p}=\operatorname{ord}_{p}(B)$, while (3.2.1) is the definition of $\mu_{\vec{v}}$.

More generally, if $G$ is any effective $\mathbb{Q}$-divisor on $S$, denote the order of $h^{*} G$ along $F_{\vec{v}}$ by $o_{\vec{v}}(G) ; o_{\vec{v}}(G)=\operatorname{ord}_{p}(G)+\operatorname{ord}_{V}\left(f^{-1} G\right)$. For convenience, let $o_{V}(G) \stackrel{\text { def }}{=} \operatorname{ord}_{V}\left(f^{-1} G\right)$, and let $\mu_{V}=o_{V}(B)$.

Note that, in general, $o_{\vec{v}}=\operatorname{ord}_{p}+o_{V}$ and $o_{V} \leq \operatorname{ord}_{p}$; in particular:

$$
\mu_{p} \leq \mu_{\vec{v}} \leq 2 \mu_{p}
$$

(3.3) Consider again $(S, B, M)$ as in $\S 1$, and fix $p, \vec{v}$ as in (3.2). Since the proofs will now be more complex, we will state the criteria for separating $\vec{v}$ at $p$ one by one, in increasing order of difficulty.

The first (and easiest) case is:

Proposition 5. (Case 1) If $\mu_{p} \geq 3$ or $\mu_{\vec{v}} \geq 4$, then $\left|K_{S}+B+M\right|$ separates $\vec{v}$ at p. ( $M$ must still be nef and big.)

Proof. Recall that the conclusion means that the restriction map

$$
H^{0}\left(S, K_{S}+B+M\right) \rightarrow H^{0}\left(Z, K_{S}+B+\left.M\right|_{Z}\right) \cong \mathcal{O}_{Z}
$$

is surjective. 
If $\mu_{p} \geq 3$, we use the vanishing theorem in the form $(1.2 .1)(\mathrm{a})$ for

$$
\begin{aligned}
K_{S_{1}}+\left\lceil f^{*} M\right\rceil & =f^{*}\left(K_{S}+B+M\right)+E_{p}-\left\lfloor f^{*} B\right\rfloor \\
& =f^{*}\left(K_{S}+B+M\right)-t E_{p}
\end{aligned}
$$

where $t=\left\lfloor\mu_{p}\right\rfloor-1 \geq 2$, as in (1.4)-(1.5); then $H^{0}\left(S, K_{S}+B+M\right) \rightarrow \mathcal{O}_{S} / \mathfrak{m}_{p}{ }^{t}$ is surjective, and since $t \geq 2$, we have $\mathfrak{m}_{p}{ }^{t} \subset \mathcal{I}_{Z}$, i.e. $\mathcal{O}_{S} / \mathfrak{m}_{p}{ }^{t} \rightarrow \mathcal{O}_{Z}$ is also surjective. (See also Remark 1.5.3.)

If $\mu_{\vec{v}} \geq 4$, the argument is similar, starting on $S_{2}$ :

$$
\begin{aligned}
K_{S_{2}}+\left\lceil h^{*} M\right\rceil & =h^{*}\left(K_{S}+B+M\right)+F_{p}+2 F_{\vec{v}}-\left\lfloor h^{*} B\right\rfloor \\
& =h^{*}\left(K_{S}+B+M\right)-t_{p} F_{p}-t_{\vec{v}} F_{\vec{v}}
\end{aligned}
$$

where $t_{\vec{v}}=\left\lfloor\mu_{\vec{v}}\right\rfloor-2 \geq 2$, and $t_{p}=\left\lfloor\mu_{p}\right\rfloor-1 \geq 1$ (indeed, by (3.2.2), $\mu_{p} \geq \frac{1}{2} \mu_{\vec{v}} \geq 2$.) As in the previous case, we get a vanishing $H^{1}\left(S, \mathcal{O}_{S}\left(K_{S}+\right.\right.$ $B+M) \otimes \mathcal{I})=0$ for $\mathcal{I}=h_{*} \mathcal{O}_{S_{2}}\left(-t_{p} F_{p}-t_{\vec{v}} F_{\vec{v}}\right) ; \operatorname{Supp}\left(\mathcal{O}_{S} / \mathcal{I}\right)=\{p\}$ and $\mathcal{I} \subset \mathcal{I}_{Z}$, so the conclusion follows as before.

(3.4) Now assume that $\mu_{p}<3$ and $\mu_{\vec{v}}<4$.

First consider the case $2 \leq \mu_{p}<3$. Then $2 \leq \mu_{\vec{v}}<4$, and therefore $0<\left(4-\mu_{\vec{v}}\right) \leq 2$.

Proposition 5. (Case 2) Let $2 \leq \mu_{p}<3$ and $2 \leq \mu_{\vec{v}}<4$. Assume that $M^{2}>\left(4-\mu_{\vec{v}}\right)^{2}, M \cdot C \geq \frac{1}{2}\left(4-\mu_{\vec{v}}\right)$ for every curve $C \subset S$ through $p$, and $M \cdot C \geq\left(4-\mu_{\vec{v}}\right)$ for every curve $C$ containing $Z-i . e$. , such that $p \in C$ and $\vec{v} \in T_{p}(C)$. Then $\left|K_{S}+B+M\right|$ separates $\vec{v}$ at $p$.

\section{Proof.}

(3.5) Claim. We can find an effective $\mathbb{Q}$-divisor $D$ on $S$ such that $o_{\vec{v}}(D)=4-\mu_{\vec{v}}$ and $D \underset{\mathbb{Q}}{\sim} t M$ for some $t \in \mathbb{Q}, 0<t<1$. (See (3.2) for the definition of $o_{\vec{v}}(D)$.)

Proof of (3.5). Choose $a>\left(4-\mu_{\vec{v}}\right)$ such that $M^{2}>a^{2}$. Then $\left(h^{*} M-\right.$ $\left.a F_{\vec{v}}\right)^{2}=M^{2}-a^{2}>0$ and $\left(h^{*} M-a F_{\vec{v}}\right) \cdot h^{*} M=M^{2}>0$; therefore $h^{*} M-a F_{\vec{v}} \in N\left(S_{2}\right)^{+}$, the positive cone of $S_{2}$, and in particular it is big. (See, for example, [KM, (1.1)].) Therefore $\exists T$, effective $\mathbb{Q}$-divisor on $S_{2}$, such that $T \underset{\mathbb{Q}}{\sim} h^{*} M-a F_{\vec{v}}$. Put $D_{1}=h_{*}\left(T+a F_{\vec{v}}\right)$; then $D_{1} \underset{\mathbb{Q}}{\sim} h_{*}\left(h^{*} M\right)=$ M. Also, $h^{*} D_{1}=T+a F_{\vec{v}}$ (their difference has support contained in $F_{p} \cup F_{\vec{v}}$; 
on the other hand, $T+a F_{\vec{v}} \underset{\mathbb{Q}}{\sim} h^{*} M \Longrightarrow\left(h^{*} D_{1}-\left(T+a F_{\vec{v}}\right)\right) \cdot F_{p}=\left(h^{*} D_{1}-(T+\right.$ $\left.\left.a F_{\vec{v}}\right)\right) \cdot F_{\vec{v}}=0$, and $h^{*} D_{1}=T+a F_{\vec{v}}$ follows from the negative definiteness of the intersection form on $\left.h^{-1}(p)=F_{p} \cup F_{\vec{v}}\right)$. We have $D_{1} \underset{\mathbb{Q}}{\sim} M$ and $o_{\vec{v}}\left(D_{1}\right) \geq a>4-\mu_{\vec{v}}$. Take $D=t D_{1}, t=\frac{4-\mu_{\vec{v}}}{o_{\vec{v}}\left(D_{1}\right)}<1$.

Remark. The statement of (3.5) is similar to that of (1.6.1), and indeed, we could have proved it as in $\S 1$. However, the proof we gave here is easier to generalize, especially on singular surfaces.

(3.6) We return to the proof of proposition 5, case 2. Choose $D$ as in (3.5). Write $B=\sum b_{i} C_{i}, D=\sum d_{i} C_{i} ; D_{i}=f^{-1} C_{i}, T_{i}=g^{-1} D_{i}=$ $h^{-1} C_{i} ; h^{*} B=h^{-1} B+\mu_{p} F_{p}+\mu_{\vec{v}} F_{\vec{v}}, h^{*} D=h^{-1} D+m_{p} F_{p}+\left(4-\mu_{\vec{v}}\right) F_{\vec{v}}$, where $m_{p}=\operatorname{ord}_{p}(D)$. We have $K_{S_{2}}=h^{*} K_{S}+F_{p}+2 F_{\vec{v}}$.

If $b_{i}+d_{i} \leq 1$ for every $C_{i}$ through $p$, then

$$
\begin{aligned}
& K_{S_{2}}+\left\lceil h^{*}(M-D)\right\rceil \\
& \quad=h^{*}\left(K_{S}+B+M\right)+F_{p}+2 F_{\vec{v}}-\left\lfloor h^{*}(B+D)\right\rfloor \\
& \quad=h^{*}\left(K_{S}+B+M\right)-t_{p} F_{p}-2 F_{\vec{v}}-\sum^{\prime} T_{i}-N_{2},
\end{aligned}
$$

where $\sum^{\prime} T_{i}$ extends over all $i$ with $b_{i}+d_{i}=1$ and $p \in C_{i}$ (if any), $N_{2}$ is an effective divisor on $S_{2}$ such that $\operatorname{Supp}\left(N_{2}\right) \cap h^{-1}(p)=\emptyset$, and $t_{p}=$ $\left\lfloor\mu_{p}+m_{p}\right\rfloor-1 \geq 1$ (because $\mu_{p} \geq 2$ by hypothesis). Then we conclude as in (3.3) (case 1 of the proposition), using the vanishing (1.2.1)(b) to dispose of $\sum^{\prime} T_{i}$ (if it is not zero).

(3.7) Now assume that $b_{i}+d_{i}>1$ for at least one $C_{i}$ through $p$. Let

$$
c \stackrel{\text { def }}{=} \min \left\{\frac{3-\mu_{p}}{m_{p}} ; \frac{1-b_{i}}{d_{i}}: b_{i}+d_{i}>1 \text { and } p \in C_{i}\right\} .
$$

If $c=\frac{3-\mu_{p}}{m_{p}}$, we finish again as in case 1, using (1.2.1)(b) for

$$
K_{S_{1}}+\left\lceil f^{*}(M-c D)\right\rceil=f^{*}\left(K_{S}+B+M\right)-2 E_{p}-\sum^{\prime} D_{i}-N_{1}
$$

on $S_{1}$, where $\sum^{\prime} D_{i}$ extends over all $i$ such that $b_{i}+c d_{i}=1$ and $p \in C_{i}$ (if any), and $\operatorname{Supp}\left(N_{1}\right) \cap E_{p}=\emptyset$. 
(3.8) If $c=\frac{1-b_{0}}{d_{0}}<\frac{3-\mu_{p}}{m_{p}}$ for some $C_{0}$ through $p$, then

$$
\sum\left(b_{i}+c d_{i}\right) \cdot \operatorname{mult}_{p}\left(C_{i}\right)=\mu_{p}+c m_{p}<3 ;
$$

therefore $\operatorname{mult}_{p}\left(C_{0}\right) \leq 2$, and moreover, if $\operatorname{mult}_{p}\left(C_{0}\right)=2$, then $b_{i}+c d_{i}<1$ for all $C_{i}$ through $p$ with $i \neq 0$. Also, $\mu_{\vec{v}}+c\left(4-\mu_{\vec{v}}\right)<4$ (since $c<1$ ), and therefore $B+c D \geq C_{0} \Longrightarrow o_{\vec{v}}\left(C_{0}\right) \leq 3$.

(3.9) If $C_{0}$ is singular at $p$ and $\vec{v} \notin T C_{p}\left(C_{0}\right)$ (the tangent cone to $C_{0}$ at $p$ ), then $o_{\vec{v}}\left(C_{0}\right)=2$. We have $Z \subset C_{0}$, and

$$
\begin{aligned}
K_{S}+\lceil M-c D\rceil & =\left(K_{S}+B+M\right)-\lfloor B+c D\rfloor \\
& =\left(K_{S}+B+M\right)-C_{0}-N,
\end{aligned}
$$

with $p \notin \operatorname{Supp}(N)$. Using (1.2.1)(a), as in $\S 1$, it suffices to show that $\left.\left(\left(K_{S}+B+M\right)-N\right)\right|_{C_{0}}$ separates $\vec{v}$ at $p$ on $C_{0}$; that, in turn, will follow from (1.2.2)(b), if we can show that $\lceil M-c D\rceil \cdot C_{0}>2$.

As before, write $\lceil M-c D\rceil=(M-c D)+\Delta ; \Delta=\{B+c D\}$ and $C_{0}$ intersect properly, and $\Delta=B+c D-C_{0}$ in an open neighborhood of $p$.

We have: $\operatorname{ord}_{p}(\Delta)=\mu_{p}+c m_{p}-2$, and therefore $\Delta \cdot C_{0} \geq 2\left(\mu_{p}+c m_{p}-2\right)$. However, we get a better estimate if we consider orders along $F_{\vec{v}}$, as follows: $o_{\vec{v}}(\Delta)=\mu_{\vec{v}}+c\left(4-\mu_{\vec{v}}\right)-2$, because $o_{\vec{v}}\left(C_{0}\right)=2 ; \operatorname{ord}_{p}(\Delta) \geq \frac{1}{2} o_{\vec{v}}(\Delta)$, and therefore

$$
\Delta \cdot C_{0} \geq \frac{1}{2} o_{\vec{v}}(\Delta) \cdot 2=\mu_{\vec{v}}+c\left(4-\mu_{\vec{v}}\right)-2 .
$$

Finally,

$$
\begin{aligned}
& \lceil M-c D\rceil \cdot C_{0} \\
& \quad=(M-c D) \cdot C_{0}+\Delta \cdot C_{0}=(1-c t) M \cdot C_{0}+\Delta \cdot C_{0} \\
& \quad \geq(1-c t)\left(4-\mu_{\vec{v}}\right)+\mu_{\vec{v}}+c\left(4-\mu_{\vec{v}}\right)-2 \\
& \quad>2 \quad \text { (because } t<1),
\end{aligned}
$$

as required.

(3.10) If $C_{0}$ is singular at $p$ and $\vec{v} \in T C_{p}\left(C_{0}\right)$, then $o_{\vec{v}}\left(C_{0}\right)=3(\geq 3$ is clear, and $\leq 3$ was shown in (3.8)).

Working as in (3.9), we can show that

$$
\lceil M-c D\rceil \cdot C_{0} \geq(1-c t)\left(4-\mu_{\vec{v}}\right)+\mu_{\vec{v}}+c\left(4-\mu_{\vec{v}}\right)-3>1
$$


(now $o_{\vec{v}}(\Delta)=o_{\vec{v}}\left(B+c D-C_{0}\right)=\mu_{\vec{v}}+c\left(4-\mu_{\vec{v}}\right)-3$ ); thus in this case we cannot use (1.2.2)(b) as in (3.9). We will modify the argument as follows:

Start with $f^{*}(M-c D)$ on $S_{1}$; the vanishing theorem yields

$$
H^{1}\left(S_{1}, f^{*}\left(K_{S}+B+M\right)-E_{p}-D_{0}-N_{1}\right)=0, \quad N_{1} \cap E_{p}=\emptyset
$$

(the coefficient of $E_{p}$ is -1 because $2 \leq \mu_{p}+c m_{p}<3$; the first inequality follows from $\mu_{p} \geq 2$, and the second was shown in (3.8)).

$\vec{v} \in T C_{p}\left(C_{0}\right) \Longrightarrow V \in D_{0}$ (recall that $V \in E_{p}$ corresponds to $\vec{v} \in$ $T_{p}(S)$ ). (3.10.2) implies the surjectivity of the restriction map

$$
\begin{aligned}
H^{0}\left(S_{1}, f^{*}\right. & \left.\left(K_{S}+B+M\right)-E_{p}-N_{1}\right) \\
& \rightarrow H^{0}\left(D_{0}, f^{*}\left(K_{S}+B+M\right)-E_{p}-\left.N_{1}\right|_{D_{0}}\right) .
\end{aligned}
$$

We will show that $\exists \tilde{\Gamma} \in\left|f^{*}\left(K_{S}+B+M\right)-E_{p}-N_{1}\right|_{D_{0}} \mid$ such that $V \notin$ $\operatorname{Supp}(\tilde{\Gamma})$. Then we can lift $\tilde{\Gamma}$ to $\Gamma \in\left|f^{*}\left(K_{S}+B+M\right)-E_{p}-N_{1}\right|$, since (3.10.3) is surjective. $\Gamma+E_{p}+N_{1} \in\left|f^{*}\left(K_{S}+B+M\right)\right|$ has the form $f^{*} \Lambda$ for some $\Lambda \in\left|K_{S}+B+M\right|$. Finally, $p \in \operatorname{Supp}(\Lambda)$, because $f^{*} \Lambda \geq E_{p}$, but $\vec{v} \notin T_{p}(\Lambda)$, because $V \notin \operatorname{Supp}\left(f^{*} \Lambda-E_{p}\right)$; this shows that $\left|K_{S}+B+M\right|$ separates $\vec{v}$ at $p$ on $S$.

To prove the existence of $\tilde{\Gamma}$, note that $\left.\left(f^{*}\left(K_{S}+B+M\right)-E_{p}-N_{1}\right)\right|_{D_{0}}=$ $K_{D_{0}}+\left.\left\lceil f^{*}(M-c D)\right\rceil\right|_{D_{0}}$; we will show that $\left\lceil f^{*}(M-c D)\right\rceil \cdot D_{0}>1$ - then (1.2.2)(a) implies the existence of $\tilde{\Gamma}$.

As in (1.9), we can write $\left\lceil f^{*}(M-c D)\right\rceil=f^{*}(M-c D)+\Delta_{1}$, where $\Delta_{1}=\left\{f^{*}(B+c D)\right\}$ and $D_{0}$ intersect properly, and $\Delta_{1}=f^{*}(B+c D)-$ $2 E_{p}-D_{0}=f^{*}\left(B+c D-C_{0}\right)$ in a neighborhood of $E_{p}$ (the coefficient of $E_{p}$ in $f^{*}(B+c D)$ is $\mu_{p}+c m_{p}$, and $\left.2 \leq \mu_{p}+c m_{p}<3\right)$. We have:

$$
\begin{aligned}
& \left\lceil f^{*}(M-c D)\right\rceil \cdot D_{0} \\
& \quad=f^{*}(M-c D) \cdot D_{0}+\Delta_{1} \cdot D_{0} \\
& \quad \geq(M-c D) \cdot C_{0}+\operatorname{ord}_{p}\left(B+c D-C_{0}\right) \cdot \operatorname{mult}_{p}\left(C_{0}\right) \\
& \quad \geq(1-c t)\left(4-\mu_{\vec{v}}\right)+\mu_{\vec{v}}+c\left(4-\mu_{\vec{v}}\right)-3 \\
& \quad>1
\end{aligned}
$$

as in (3.10.1).

(3.11) Now consider the case: $C_{0}$ smooth at $p$ and tangent to $\vec{v}$, and $b_{i}+c d_{i}<1$ for all $i \neq 0$ with $p \in C_{i}$. 
Write $\lceil M-c D\rceil=(M-c D)+\Delta$, where $\Delta$ and $C_{0}$ intersect properly; then $\Delta \cdot C_{0}=h^{*} \Delta \cdot T_{0}$ (projection formula: recall that $\left.T_{0}=h^{-1} C_{0}\right) \geq o_{\vec{v}}(\Delta)$, because $F_{\vec{v}} \cdot T_{0}=1$; since $\Delta=\{B+c D\}=B+c D-C_{0}$ in a neighborhood of $p$, we have $o_{\vec{v}}(\Delta)=\mu_{\vec{v}}+c\left(4-\mu_{\vec{v}}\right)-2$, and therefore

$$
\lceil M-c D\rceil \cdot C_{0} \geq(1-c t)\left(4-\mu_{\vec{v}}\right)+\mu_{\vec{v}}+c\left(4-\mu_{\vec{v}}\right)-2>2,
$$

exactly as in (3.9.1). Thus $K_{C_{0}}+\left.\lceil M-c D\rceil\right|_{C_{0}}$ separates $\vec{v}$ on $C_{0}$; we conclude as in (3.9).

(3.12) If $C_{0}$ is smooth at $p$ and tangent to $\vec{v}$, and moreover $b_{i}+c d_{i}=1$ for at least one $i \neq 0$ with $p \in C_{i}$, then: such an $i$ is unique, say $i=1$, and $C_{1}$ must be smooth at $p$ and not tangent to $\vec{v}$; indeed, $B+c D \geq C_{0}+C_{1}$, while $\operatorname{ord}_{p}(B+c D)<3$ and $o_{\vec{v}}(B+c D)<4$.

In this case reverse the roles of $C_{0}$ and $C_{1}$ : thus $C_{0}$ will be smooth at $p$ and not tangent to $\vec{v}$. This situation is covered below, in (3.13).

(3.13) Finally, assume that $C_{0}$ is smooth at $p$ and not tangent to $\vec{v}$. In this case we work with $M-c^{\prime} D$ for some $c^{\prime} \geq c$, namely:

$$
c^{\prime} \stackrel{\text { def }}{=} \min \left\{1 ; \frac{3-\mu_{p}}{m_{p}} ; \frac{2-b_{0}}{d_{0}} ; \frac{1-b_{i}}{d_{i}} \text { with } i \neq 0, p \in C_{i} \text { and } b_{i}+d_{i} \geq 1\right\} \text {. }
$$

In all cases, $M-c^{\prime} D \underset{\mathbb{Q}}{\sim}\left(1-c^{\prime} t\right) M$ is still nef and big; using the vanishing $H^{1}\left(S, K_{S}+\left\lceil M-c^{\prime} D\right\rceil\right)=0$, or the corresponding vanishing on $S_{1}$ or $S_{2}$, we will show that $\exists \Lambda \in\left|K_{S}+B+M-C_{0}-N\right|, p \notin \operatorname{Supp}(N)$, such that $p \notin \operatorname{Supp}(\Lambda)$. Then $\Lambda+C_{0}+N \in\left|K_{S}+B+M\right|$ has the unique component $C_{0}$ through $p$ not tangent to $\vec{v}$, as required.

It remains to prove the existence of $\Lambda$.

(3.13.1) If $c^{\prime}=\frac{3-\mu_{p}}{m_{p}}$, then (1.2.1)(b) yields

$$
H^{1}\left(S_{1}, f^{*}\left(K_{S}+B+M\right)-2 E_{p}-D_{0}-N_{1}\right)=0, \quad N_{1} \cap E_{p}=\emptyset ;
$$

thus $H^{1}\left(S_{1}, f^{*}\left(K_{S}+B+M-C_{0}-N\right)-E_{p}\right)=0$, where $N=f_{*} N_{1}$, and the existence of $\Lambda$ follows.

When $c^{\prime}=1$ the proof is similar, starting on $S_{2}$, as in the proof of case 1 of the proposition.

(3.13.2) If $c^{\prime}=\frac{1-b_{1}}{d_{1}}<\frac{3-\mu_{p}}{m_{p}}$ for another curve $C_{1}$ through $p$ with $b_{1}+d_{1}>1$, then $C_{1}$ must be smooth at $p$ (because $B+c^{\prime} D \geq C_{0}+C_{1}$, 
and $\left.\operatorname{ord}_{p}\left(B+c^{\prime} D\right)=\mu_{p}+c^{\prime} m_{p}<3\right)$. We may have $c^{\prime}=c$ (e.g., in the case discussed in (3.12)), or $c^{\prime}>c$. In any event, $b_{i}+c^{\prime} d_{i}<1$ for all curves $C_{i}$ through $p, i \neq 0,1$. (1.2.1)(a) yields:

$$
H^{1}\left(S, K_{S}+B+M-C_{0}-C_{1}-N\right)=0, \quad p \notin \operatorname{Supp}(N) .
$$

We claim that $p \notin \mathrm{Bs}\left|K_{S}+B+M-C_{0}-N\right|_{C_{1}} \mid$, which in turn follows from (1.2.2)(a) once we show that $\left\lceil M-c^{\prime} D\right\rceil \cdot C_{1}>1$. Then we use (3.13.3) to lift from $C_{1}$ to $S$, proving the existence of $\Lambda$ as stated.

$$
\left\lceil M-c^{\prime} D\right\rceil=\left(M-c^{\prime} D\right)+\Delta^{\prime}, \text { with } \Delta^{\prime}=\left\{B+c^{\prime} D\right\}=B+c^{\prime} D-C_{0}-C_{1}
$$
in a neighborhood of $p$, and $\Delta^{\prime}, C_{1}$ intersect properly.

If $\vec{v} \notin T_{p}\left(C_{1}\right)$, then $M \cdot C_{1} \geq \frac{1}{2}\left(4-\mu_{\vec{v}}\right)$ by hypothesis, and $\operatorname{ord}_{p}\left(\Delta^{\prime}\right) \geq$ $\frac{1}{2} o_{\vec{v}}\left(\Delta^{\prime}\right)=\frac{1}{2}\left(\mu_{\vec{v}}+c^{\prime}\left(4-\mu_{\vec{v}}\right)-2\right)$; therefore

$$
\left\lceil M-c^{\prime} D\right\rceil \cdot C_{1} \geq \frac{1}{2}\left(1-c^{\prime} t\right)\left(4-\mu_{\vec{v}}\right)+\frac{1}{2}\left(\mu_{\vec{v}}+c^{\prime}\left(4-\mu_{\vec{v}}\right)-2\right)>1,
$$

as required (compare to (3.9.1)). The proof is the same when $c^{\prime}=\frac{2-b_{0}}{d_{0}}$, i.e. $C_{1}=C_{0}$; in that case $p \notin \mathrm{Bs}\left|K_{S}+B+M-C_{0}-N\right|_{C_{0}} \mid$.

If $\vec{v} \in T_{p}\left(C_{1}\right)$, then $M \cdot C_{1} \geq 4-\mu_{\vec{v}}$ and $\Delta^{\prime} \cdot C_{1} \geq o_{\vec{v}}\left(\Delta^{\prime}\right)=\mu_{\vec{v}}+c^{\prime}(4-$ $\left.\mu_{\vec{v}}\right)-3$; all told, we have

$$
\left\lceil M-c^{\prime} D\right\rceil \cdot C_{1} \geq\left(1-c^{\prime} t\right)\left(4-\mu_{\vec{v}}\right)+\mu_{\vec{v}}+c^{\prime}\left(4-\mu_{\vec{v}}\right)-3>1,
$$

as claimed.

This concludes the proof of proposition 5 , case 2 .

(3.14) Finally, consider the case $0 \leq \mu_{p}<2$ (and therefore $0 \leq \mu_{V}<2$ and $\left.0 \leq \mu_{\vec{v}}=\mu_{p}+\mu_{V}<4\right)$.

Proposition 5. (Case 3) Assume that $0 \leq \mu_{p}<2$. Assume, moreover, that $M^{2}>\left(\beta_{2, p}\right)^{2}+\left(\beta_{2, V}\right)^{2}$ and

(i) $M \cdot C \geq \beta_{1}$ for every curve $C \subset S$ passing through $p$,

(ii) $M \cdot C \geq 2 \beta_{1}$ for every curve $C$ containing $Z$ (i.e., passing through $p$ and with $\vec{v} \in T_{p}(C)$ ),

where $\beta_{2, p}, \beta_{2, V}, \beta_{1}$ are positive numbers which satisfy:

$$
\begin{aligned}
& \beta_{2, p} \geq 2-\mu_{p}, \quad \beta_{2, V} \geq 2-\mu_{V} ; \\
& \beta_{1} \geq \min \left\{\frac{1}{2}\left(4-\mu_{\vec{v}}\right) ; \frac{\beta_{2, p}+\beta_{2, V}}{\beta_{2, p}+\beta_{2, V}-\left(2-\mu_{\vec{v}}\right)}\right\}
\end{aligned}
$$


Proof. The proof is very similar, in many respects, to that of case 2 . We indicate the main steps of the proof, and we provide explicit computations in a few cases, to show what kind of alterations are needed.

(3.15) Claim. We can find $D$, an effective $\mathbb{Q}$-divisor on $S$, such that $o_{\vec{v}}(D)=4-\mu_{\vec{v}}$ and $D \underset{\mathbb{Q}}{\sim} t M$ for some $t \in \mathbb{Q}, t>0$, satisfying

$$
t<\frac{4-\mu_{\vec{v}}}{\beta_{2, p}+\beta_{2, V}}
$$

- and therefore, in particular, $t<1$.

Proof of (3.15). Choose $a>\beta_{2, p}, b>\beta_{2, V}$, such that $M^{2}>a^{2}+b^{2}$. We have $\left(a F_{p}+(a+b) F_{\vec{v}}\right)^{2}=-\left(a^{2}+b^{2}\right)$, and therefore $h^{*} M-\left(a F_{p}+(a+b) F_{\vec{v}}\right)$ is big, as in the proof of (3.5). Thus we can find $D_{1} \underset{\mathbb{Q}}{\sim} M$ on $S, D_{1} \geq 0$, such that $h^{*} D_{1} \geq a F_{p}+(a+b) F_{\vec{v}}$. Then take $D=t D_{1}$, with

$$
t=\frac{4-\mu_{\vec{v}}}{o_{\vec{v}}\left(D_{1}\right)} \leq \frac{4-\mu_{\vec{v}}}{a+b}<\frac{4-\mu_{\vec{v}}}{\beta_{2, p}+\beta_{2, V}} .
$$

(3.16) If $D=\sum d_{i} C_{i}$, as before, and $b_{i}+d_{i} \leq 1$ for every $C_{i}$ through $p$, we conclude as in (3.6).

If $b_{i}+d_{i}>1$ for at least one $C_{i}$ through $p$, then define

$$
c=\min \left\{\frac{3-\mu_{p}}{m_{p}} ; \frac{1-b_{i}}{d_{i}}: b_{i}+d_{i}>1 \text { and } p \in C_{i}\right\} .
$$

If $c=\frac{3-\mu_{p}}{m_{p}}$, we finish as in (3.7).

If $c=\frac{1-b_{0}}{d_{0}}<\frac{3-\mu_{p}}{m_{p}}$ for some $C_{0}$ through $p$, then $\operatorname{mult}_{p}\left(C_{0}\right) \leq 2$ and $o_{\vec{v}}\left(C_{0}\right) \leq 3$; if $C_{0}$ is singular at $p$, then it is the only $C_{i}$ through $p$ with $b_{i}+c d_{i} \geq 1$, and we proceed as in (3.9) or (3.10), according to whether $\vec{v} \in T C_{p}\left(C_{0}\right)$ or not. Only the proof of $\lceil M-c D\rceil \cdot C_{0}>2$ (if $\vec{v} \notin T C_{p}\left(C_{0}\right)$ ) or $>1$ (if $\vec{v} \in T C_{p}\left(C_{0}\right)$ ) needs adjustment.

Assume first that $\vec{v} \notin T C_{p}\left(C_{0}\right)$ (with $C_{0}$ singular at $p$ ). Then $\lceil M-$ $c D\rceil=(M-c D)+\Delta, \Delta=\{B+c D\}=B+c D-C_{0}$ in a neighborhood of $p$, and $o_{\vec{v}}(\Delta)=\mu_{\vec{v}}+c\left(4-\mu_{\vec{v}}\right)-2 ; \operatorname{ord}_{p}(\Delta) \geq \frac{1}{2} o_{\vec{v}}(\Delta)$ and $\operatorname{mult}_{p}\left(C_{0}\right)=2$, so that

$$
\lceil M-c D\rceil \cdot C_{0} \geq(1-c t)\left(2 \beta_{1}\right)+\mu_{\vec{v}}+c\left(4-\mu_{\vec{v}}\right)-2 .
$$


If $\beta_{1} \geq \frac{1}{2}\left(4-\mu_{\vec{v}}\right)$, then $\lceil M-c D\rceil \cdot C_{0}>2$ follows from (3.16.2) and $t<1$. In particular, this is true if $\mu_{\vec{v}} \geq 2$. If $\mu_{\vec{v}}<2$, the hypothesis is weaker than $\beta_{1} \geq \frac{1}{2}\left(4-\mu_{\vec{v}}\right)$, namely:

$$
\beta_{1} \geq \frac{\beta_{2, p}+\beta_{2, V}}{\beta_{2, p}+\beta_{2, V}-\left(2-\mu_{\vec{v}}\right)} .
$$

Assume also that $\beta_{1}<\frac{1}{2}\left(4-\mu_{\vec{v}}\right)$ (otherwise we are done). Then $\lceil M-c D\rceil$. $C_{0}>2$ follows from $(3.16 .2),(3.16 .3),(3.15 .1)$, and $c \geq \frac{2-\mu_{\vec{v}}}{4-\mu_{\vec{v}}}$, exactly as in (1.11).

Now consider the case $\vec{v} \in T C_{p}\left(C_{0}\right)$ (with $C_{0}$ still singular at $p$ ). Using the strategy of $(3.10)$, all we need to prove is $\lceil M-c D\rceil \cdot C_{0}>1$, which follows from

$$
\lceil M-c D\rceil \cdot C_{0} \geq(1-c t)\left(2 \beta_{1}\right)+\mu_{\vec{v}}+c\left(4-\mu_{\vec{v}}\right)-3
$$

(same computation as in (3.10) - see (3.10.4)). Using (3.16.4), the inequality $\lceil M-c D\rceil \cdot C_{0}>1$ is proved exactly as in the previous paragraph.

(3.17) If $C_{0}$ is smooth at $p, \vec{v} \in T_{p}\left(C_{0}\right)$, and $b_{i}+c d_{i}<1$ for every $C_{i}$ through $p$ with $i \neq 0$, then the proof goes as in (3.11); the inequality we need in this case, $\lceil M-c D\rceil \cdot C_{0}>2$, is proved as above.

As in the proof of case 2 of the proposition, if $C_{0}$ is smooth at $p$ and tangent to $\vec{v}$, and $b_{1}+c d_{1}=1$ for one more curve $C_{1}$ through $p$, then $C_{1}$ is unique with these properties, and is smooth at $p$ and $\vec{v} \notin T_{p}\left(C_{1}\right)$. Switching $C_{0}$ and $C_{1}$, we are in the situation discussed below. (Compare to (3.12).)

(3.18) Finally, assume that $C_{0}$ is smooth at $p$ and $\vec{v} \notin T_{p}\left(C_{0}\right)$. Define

$$
c^{\prime}=\min \left\{1 ; \frac{3-\mu_{p}}{m_{p}} ; \frac{2-b_{0}}{d_{0}} ; \frac{1-b_{i}}{d_{i}}: i \neq 0, b_{i}+d_{i}>1 \text { and } p \in C_{i}\right\} .
$$

Consider, for example, the case $c^{\prime}=\frac{2-b_{0}}{d_{0}}<1$ and $<\frac{3-\mu_{p}}{m_{p}}$. In this case, we show that $p \notin \mathrm{Bs}\left|K_{S}+B+M-C_{0}-N\right|$, for some effective divisor $N$ supported away from $p$. Using the vanishing

$$
H^{1}\left(S, K_{S}+\left\lceil M-c^{\prime} D\right\rceil\right)=H^{1}\left(S, K_{S}+B+M-2 C_{0}-N\right)=0,
$$

it suffices to show that $p \notin \operatorname{Bs}\left|K_{S}+B+M-C_{0}-N\right|_{C_{0}} \mid$; this, in turn, will follow from $(1.2 .2)(\mathrm{a})$ and $\left\lceil M-c^{\prime} D\right\rceil \cdot C_{0}>1$. 
Now $C_{0}$ passes through $p$ but is not tangent to $\vec{v}$, and therefore we have only $M \cdot C_{0} \geq \beta_{1}$ (rather than $\left.2 \beta_{1}\right)$. $\left\lceil M-c^{\prime} D\right\rceil=\left(M-c^{\prime} D\right)+\Delta^{\prime}$, with $\Delta^{\prime}=B+c^{\prime} D-2 C_{0}$ in a neighborhood of $p$, and therefore $\operatorname{ord}_{p}\left(\Delta^{\prime}\right) \geq$ $\frac{1}{2}\left(\mu_{\vec{v}}+c^{\prime}\left(4-\mu_{\vec{v}}\right)-2\right)$; it suffices to show that

$$
\left(1-c^{\prime} t\right) \beta_{1}+\frac{1}{2}\left(\mu_{\vec{v}}+c^{\prime}\left(4-\mu_{\vec{v}}\right)-2\right)>1 .
$$

An inequality equivalent to this one was already proved in (3.16).

The proof in the remaining cases is a similar adaptation of the arguments in (3.13).

\section{Example}

Fix an integer $n, n \geq 1$. Let $S$ be the $n^{\text {th }}$ Hirzebruch surface, i.e. the geometrically ruled rational surface $\mathbb{P}(\mathcal{E})$, where $\mathcal{E}$ is the rank 2 vector bundle $\mathcal{O}_{\mathbb{P}^{1}} \oplus \mathcal{O}_{\mathbb{P}^{1}}(-n)$ on $\mathbb{P}^{1}$. Let $\pi: S \rightarrow \mathbb{P}^{1}$ be the ruling of $S$, and let $F$ denote a fiber of $\pi$. $S$ contains a unique irreducible curve $G$ with negative self-intersection, $G^{2}=-n$. $\operatorname{Pic}(S) \cong \mathbb{Z} \oplus \mathbb{Z}$, with generators $F$ and $G$; $F^{2}=0, F \cdot G=1$. $K_{S} \sim-2 G-(n+2) F$. If $C$ is any irreducible curve on $S$, then $C=G, C \sim F$, or $C \sim a G+b F$ with $a, b \in \mathbb{Z}, a \geq 1$ and $b \geq n a$. All these properties are proved, for example, in [Har1, Ch.V, §2].

Let $H_{m}=G+m F$. We will use the Reider-type results for $\mathbb{Q}$-divisors to prove the following facts:

Claim. (1) (See [Bv, Ch.IV, Ex.1].) $\left|H_{n}\right|$ is base-point-free, and defines a morphism $\phi_{n}: S \rightarrow \mathbb{P}^{n+1}$. Moreover, $\phi_{n}$ is an isomorphism on $S \backslash G$, and $\bar{S}=\phi_{n}(S) \subset \mathbb{P}^{n+1}$ is a (projective) cone with vertex $x=\phi_{n}(G)$. (Thus $\bar{S}$ is the cone over a normal rational curve contained in a hyperplane $\mathbb{P}^{n} \subset \mathbb{P}^{n+1}$, because $G \cong \mathbb{P}^{1}$ and $G^{2}=-n$. $\phi_{n}$ is the blowing-up of $\bar{S}$ at $x$.)

(2) $\left|H_{m}\right|$ is very ample for $m \geq n+1$, defining an embedding $\phi_{m}: S \rightarrow$ $\mathbb{P}^{2 m-n+1}$. (See [Bv, Ch.IV, Ex.2] for other properties of $\left|H_{m}\right|$.)

Certainly, these facts can be proved in many different ways. For instance, for (1): if $G \subset S$ is a smooth rational curve with negative selfintersection on any smooth surface $S$, then there is a projective contraction $\phi: S \rightarrow \bar{S}$, which is an isomorphism on $S \backslash G$ and contracts $G$ to a normal point $x$. (This is a direct generalization of the "easy" part of Castelnuovo's criterion - the "hard" part being the regularity of $\bar{S}$ at $x$ when $G^{2}=-1$.) The proof can be adapted to the situation of the Claim. Alternatively, most of the Claim is proved in [Har1, Ch.V, Theorem 2.17]. 
The methods used in these proofs are somewhat specialized (the "normal contraction" approach depends on $\operatorname{Pic}(G) \cong \mathbb{Z}$; the proof in [Har1] is typical for ruled surfaces). From this point of view, Reider's theorem, which is based only on intersection numbers, is much more general. However, as we will see, Reider's theorem doesn't apply in the situation of the Claim. The proof we give below shows that there are instances where the scope of Reider's original results can be broadened by allowing $\mathbb{Q}$-divisors into the picture.

\section{Proof of the Claim}

(1) Write $H_{n}=K_{S}+L$, thus defining $L=H_{n}-K_{S}=3 G+(2 n+2) F$. Then $L \cdot G=3(-n)+(2 n+2)=-n+2$; thus $L$ is not nef for $n \geq 3$, and therefore Reider's criterion does not apply. However, write $L$ as $B+M$, with $B=(1-\epsilon) G$ and $M=(2+\epsilon) G+(2 n+2) F, \epsilon \in(0,1)$. Then

$$
M \cdot F=(2+\epsilon), \quad M \cdot G=(2-\epsilon n), \quad M^{2}=(2+\epsilon)(2 n+4-\epsilon n) .
$$

In particular, for $\epsilon \rightarrow 0$, we have $M \cdot F \rightarrow 2, M \cdot G \rightarrow 2, M^{2} \rightarrow 2(2 n+4) \geq 12$. Fix $\epsilon>0, \epsilon \ll 1$, such that $M^{2}>9, M \cdot F \geq \frac{3}{2}$, and $M \cdot G \geq \frac{3}{2}$. Since any irreducible curve $C \subset S$ is either $C=G$, or $C \sim F$, or $C \sim a G+b F$ with $a \geq 1$ and $b \geq n a$, we automatically have $M \cdot C \geq \frac{3}{2}$ for all such $C$. (We will use this observation again later: if $M \cdot G \geq 0$ and $C \neq G$ is an irreducible curve, then $M \cdot C \geq M \cdot F$.) Therefore $\left|H_{n}\right|$ is base-point-free by Proposition 3, part 2, with $\beta_{2}=3$ and $\beta_{1}=\frac{3}{2}=\frac{\beta_{2}}{\beta_{2}-1}$.

Thus $\left|H_{n}\right|$ defines a morphism $\phi_{n}: S \rightarrow \mathbb{P}^{\nu}, \nu=\operatorname{dim}\left|H_{n}\right|$. We compute $\nu$. By Riemann-Roch, we have:

$$
\chi\left(S, H_{n}\right)=\frac{H_{n} \cdot\left(H_{n}-K_{S}\right)}{2}+\chi\left(S, \mathcal{O}_{S}\right)=n+2 .
$$

We get $\nu=h^{0}\left(S, H_{n}\right)-1=n+1$, as stated in the Claim, if we can show that $h^{i}\left(S, H_{n}\right)=0$ for $i \geq 1$. If we write $H_{n}=K_{S}+L$, as before, Kodaira's vanishing theorem does not apply, because $L$ is not ample (it is not even nef). If we write $L=B+M$ as above, though, we get $h^{i}\left(S, H_{n}\right)=0$ for $i \geq 1$, by $(1.2 .1)(\mathrm{a})$.

Next we show that $\phi_{n}$ is an isomorphism on $S \backslash G$. Consider two distinct points $p, q \in F \backslash G$. Write $L=B^{\prime}+M^{\prime}$, with $B^{\prime}=(1-\epsilon) G+(1-$ $\alpha) F, M^{\prime}=(2+\epsilon) G+(2 n+1+\alpha) F, \epsilon, \alpha \in(0,1)$. (Note that we may use any decomposition of $L$ of the form $B+M$, as long as $\lceil M\rceil=L$.) We have:

$$
M^{\prime} \cdot F=(2+\epsilon), \quad M^{\prime} \cdot G=(1+\alpha-\epsilon n),
$$




$$
\left(M^{\prime}\right)^{2}=(2+\epsilon)(2 n+2+2 \alpha-\epsilon n)
$$

In particular, for $\epsilon, \alpha \rightarrow 0, M^{\prime}$ is nef and big and $M^{\prime} \rightarrow 2(2 n+2) \geq 8$. Let $\mu \stackrel{\text { def }}{=} \mu_{p}=\mu_{q}=1-\alpha$. Choose $\beta_{2}=\beta_{2, p}=\beta_{2, q}=\frac{3}{2}$ (say); then $\beta_{2} \geq 2-\mu=1+\alpha$ and $\left(M^{\prime}\right)^{2}>2\left(\beta_{2}\right)^{2}$ for $\epsilon, \alpha \ll 1$.

Fix $\epsilon \ll 1$, and then choose $\alpha \ll \epsilon$ such that $1+\frac{\epsilon}{2} \geq \frac{\beta_{2}}{\beta_{2}-(1-\mu)}=\frac{\beta_{2}}{\beta_{2}-\alpha}$; this can be done, because $\frac{\beta_{2}}{\beta_{2}-\alpha} \rightarrow 1$ for $\alpha \rightarrow 0$. Then $M^{\prime} \cdot F=2+\epsilon=2 \beta_{1}$, with $\beta_{1}=1+\frac{\epsilon}{2}$ - and therefore $M^{\prime} \cdot C \geq 2 \beta_{1}$ for every irreducible curve $C$ through $p$ or $q$. Hence proposition 4 , part 3 , applies (with $\beta_{1, p}=\beta_{1, q}=\beta_{1}$ ): $\left|H_{n}\right|$ separates $(p, q)$.

If $p, q \in S \backslash G$ are distinct points on another irreducible curve $\bar{F} \sim F$, the proof is similar - take $B^{\prime}=(1-\epsilon) G+(1-\alpha) \bar{F}$. (We say $\bar{F} \sim F$ instead of "fiber of $\pi: S \rightarrow \mathbb{P}^{1}$ ", to emphasize that the proof uses numerical arguments only.) Finally, if no such curve passes through both $p$ and $q$, the proof is even easier.

Separation of tangent directions on $S \backslash G$ is proved exactly the same way; note that $\mu_{p}\left(B^{\prime}\right)=\mu_{V}\left(B^{\prime}\right)=1-\alpha$ if $B^{\prime}=(1-\epsilon) G+(1-\alpha) F, p \in$ $F \backslash G$, and $\vec{v} \in T_{p}(F) \backslash\{\overrightarrow{0}\}$.

$H_{n} \cdot G=0$ and $H_{n} \cdot F=1$; therefore $\phi_{n}$ contracts $G$ to a point $x \in$ $\bar{S}=\phi_{n}(S) \subset \mathbb{P}^{n+1}$, and $\phi_{n}(\bar{F})$ is a straight line in $\mathbb{P}^{n+1}$ for every $\bar{F} \sim F$.

(2) As in part (1) of the Claim, we can show that $\left|H_{m}\right|$ is base-pointfree for $m \geq n+1$, and defines a morphism $\phi_{m}: S \rightarrow \mathbb{P}^{2 m-n+1}$ which is an isomorphism on $S \backslash G$. For $m \geq n+1$, we must show that $\left|H_{m}\right|$ separates $p, q$ even when $p$ (or $q$, or both) is on $G$, and also that $\left|H_{m}\right|$ separates tangent directions at every point $p \in G$.

Let $\{p\}=F \cap G$ and $\vec{v} \in T_{p}(G) \backslash\{\overrightarrow{0}\}$. We will show that $\left|H_{n+1}\right|$ separates $\vec{v}$ at $p$; the other properties have similar proofs.

Write $H_{n+1}=K_{S}+L, L=3 G+(2 n+3) F$. Write $L=B+M, B=$ $(1-\epsilon) G, M=(2+\epsilon) G+(2 n+3) F, \epsilon \in(0,1)$. We have:

$$
M \cdot F=(2+\epsilon), \quad M \cdot G=(3-\epsilon n), \quad M^{2}=(2+\epsilon)(2 n+6-\epsilon n) .
$$

For $\epsilon \rightarrow 0$ we have $M \cdot F \rightarrow 2, M \cdot G \rightarrow 3$, and $M^{2} \rightarrow 2(2 n+6) \geq 16$; in particular $M$ is nef and big. (Note that $L$ itself is not nef, if $n \geq 4$; indeed, $L \cdot G=3-n$.) We have $M \cdot C \geq 2+\epsilon$ for every irreducible curve $C \subset S$ (assuming that $\epsilon \ll 1$ ); also, if $\vec{v} \in T_{p}(C)$, then $M \cdot C \geq 3-\epsilon n$, because in that case $C \sim a G+b F$ with $a \geq 1$ (proof: if $C \neq G$, then $C \cdot G \geq 2$, because $\vec{v} \in T_{p} C \cap T_{p} G$; therefore $C \not F$.) 
We have $\mu_{p}=\mu_{V}=1-\epsilon$, and $\mu_{\vec{v}}=2(1-\epsilon)$. Choose $\beta_{2}=\beta_{2, p}=$ $\beta_{2, V}=2$ (say), so that $M^{2}>2\left(\beta_{2}\right)^{2}, \beta_{2, p} \geq 2-\mu_{p}$, and $\beta_{2, V} \geq 2-\mu_{V}$. Put $\beta_{1}=\frac{2 \beta_{2}}{2 \beta_{2}-\left(2-\mu_{\vec{v}}\right)}=\frac{\beta_{2}}{\beta_{2}-\epsilon}$. For $\epsilon \ll 1$, we have:

$M \cdot C=2+\epsilon \geq \beta_{1} \quad$ for all curves $C \subset S$,

$M \cdot C=3-\epsilon n \geq 2 \beta_{1} \quad$ for all $C$ containing $(p, \vec{v})$.

(Note that $\beta_{1}=\frac{\beta_{2}}{\beta_{2}-\epsilon} \rightarrow 1$ as $\epsilon \rightarrow 0$, so these inequalities are verified for all $\epsilon \ll 1$.) Now use proposition 5 , case 3 .

By inspecting the proof of the Claim, we can see that the only assumptions we used were that $\operatorname{Pic}(S)=\mathbb{Z} G \oplus \mathbb{Z} F, G^{2}=-n, F^{2}=0, G \cdot F=$ 1 , and $K_{S}=-2 G-(n+2) F$ (if the other hypotheses are satisfied, the last condition is equivalent to: $G$ and $F$ are smooth rational curves); this suggests the following

ExERCISE. A surface $S$ with these properties is isomorphic to the $n^{\text {th }}$ Hirzebruch surface.

Hint. There are several ways to see this. One, of course, is to use part (1) of the Claim: after all, we have shown that $S$ is the blowing-up of the cone over the normal rational curve of degree $n$.

Another solution is to show that $|F|$ is base-point-free and $\operatorname{dim}|F|=1$, as in the proof of part (1) of the Claim; thus $\phi=\phi_{|F|}$ realizes $S$ as a geometrically ruled surface over $\mathbb{P}^{1}$, as required. ( $S$ is minimal, because $C^{2} \geq 0$ for every irreducible curve $C \neq G$; this follows easily from the hypotheses.)

\section{REFERENCES}

[Bv] A. Beauville, Surfaces algébriques complexes, Astérisque, 54, 1978.

[EL] L. Ein and R. Lazarsfeld, Global generation of pluricanonical and adjoint linear series on smooth projective threefolds, J. Amer. Math. Soc., 6 (1993), 875-903.

[Fuj] T. Fujita, contribution to Birational geometry of algebraic varieties: Open problems, the $23^{\text {rd }}$ Int. Symposium of the Division of Math. of the Taniguchi Foundation, Katata, August 1988.

[Har1] R. Hartshorne, Algebraic Geometry, GTM 52, Springer-Verlag, New-York, 1978.

[Har2] R. Hartshorne, Generalized divisors on Gorenstein curves and a theorem of Noether, J. Math. Kyoto Univ., 26 (1986), 375-386.

[KM] T. Kawachi and V. Maşek, Reider-type theorems on normal surfaces, J. Algebraic Geometry, 7 (1998), 239-249. 
[Kaw] Y. Kawamata, On Fujita's freeness conjecture for 3-folds and 4-folds, Math. Ann., 308 (1997), 491-505.

[KMM] Y. Kawamata, K. Matsuda and K. Matsuki, Introduction to the minimal model program, in Algebraic Geometry, Sendai 1985, Adv. Stud. Pure Math., 10 (1987), 283-360.

[Rei] I. Reider, Vector bundles of rank 2 and linear systems on algebraic surfaces, Ann. of Math., 127 (1988), 309-316.

Department of Mathematics

Box 1146

Washington University

St. Louis, MO 63130

vmasek@math. wustl. edu 